\title{
A post-transcriptional program of chemoresistance by AU-rich elements and TTP
}

Sooncheol Lee ${ }^{1,2,3,4}$, Douglas Micalizzi ${ }^{1,2}$, Samuel S Truesdell ${ }^{1,3,4}$, Syed IA Bukhari ${ }^{1,2,3,4}$, Myriam Boukhali $^{1,2}$, Jennifer Lombardi-Story ${ }^{1,2}$, Yasutaka Kato ${ }^{5}$, Min-Kyung Choo ${ }^{6}$, Ipsita Dey-Guha ${ }^{1,2}$, Benjamin T. Nicholson ${ }^{1}$, David T. Myers ${ }^{1}$, Dongjun Lee ${ }^{7}$, Maria A Mazzola ${ }^{8}$, Radhika Raheja ${ }^{8}$, Adam Langenbucher $^{1,9}$, Nicholas J. Haradhvala ${ }^{1,9,10}$, Michael Lawrence ${ }^{1,9,10}$, Roopali Gandhi ${ }^{8}$, Christopher Tiedje $^{11}$, Manuel Diaz-Munoz ${ }^{12}$, David A Sweetser ${ }^{1,13}$, David Sykes ${ }^{2,3,4}$, Wilhelm Haas ${ }^{1,2}$, Daniel A. Haber $^{1,2,14}$, Shyamala Maheswaran ${ }^{1,15}$, and Shobha Vasudevan ${ }^{1,2,3,4 *}$

${ }^{1}$ Massachusetts General Hospital Cancer Center, Harvard Medical School, Boston, MA 02114

${ }^{2}$ Department of Medicine, Massachusetts General Hospital and Harvard Medical School, Boston, MA

${ }^{3}$ Center for Regenerative Medicine, Massachusetts General Hospital, Harvard Medical School, Boston, MA 02114

${ }^{4}$ Harvard Stem Cell Institute, Harvard University, Cambridge, MA 02138

${ }^{5}$ Laboratory of Oncology, Hokuto Hospital, Obihiro, Japan

${ }^{6}$ Cutaneous Biology Research Center, Massachusetts General Hospital and Harvard Medical School, Charlestown, MA 02129

${ }^{7}$ Department of Medicine, Pusan National University School of Medicine, South Korea

${ }^{8}$ Center for Neurological Diseases, Brigham \& Women's Hospital, Harvard Medical School, Boston, MA 02115

${ }^{9}$ Department of Pathology, Massachusetts General Hospital, Harvard Medical School, Charlestown, MA 02129

${ }^{10}$ Broad Institute of Harvard \& MIT, Cambridge, MA 02142

${ }^{11}$ Department of Cellular and Molecular Medicine, Center for Healthy Aging, University of Copenhagen, Blegdamsvej 3B, 2200 Copenhagen, Denmark.

${ }^{12}$ Centre de Physiopathologie Toulouse-Purpan, INSERM UMR1043/CNRS U5282, Toulouse, France

${ }^{13}$ Department of Pediatrics, Divisions of Pediatric Hematology/Oncology and Medical Genetics, Massachusetts General Hospital, Harvard Medical School, Boston, MA 02114

${ }^{14}$ Howard Hughes Medical Institute, Chevy Chase, MD 20815

${ }^{15}$ Department of Surgery, Massachusetts General Hospital and Harvard Medical School, Charlestown, MA 02129

*Correspondence \& contact: Shobha Vasudevan 185 Cambridge St, CPZN4202, Boston MA 02114; Ph: 617-643-3143; vasudevan.shobha@mgh.harvard.edu

Running title: Chemoresistance by AU-rich elements and TTP

Notes 3990 words; 8 main \& 6 supplemental figures 


\section{Abstract}

Background: Quiescence (G0) is a transient, cell cycle-arrested state. By entering G0, cancer cells survive unfavorable conditions such as chemotherapy and cause relapse. While G0 cells have been studied at the transcriptome level, how post-transcriptional regulation contributes to their chemoresistance remains unknown.

Results: We induced chemoresistant and quiescent (G0) leukemic cells by serum-starvation or chemotherapy treatment. To study post-transcriptional regulation in G0 leukemic cells, we systematically analyzed their transcriptome, translatome, and proteome. We find that our resistant G0 cells recapitulate gene expression profiles of in vivo chemoresistant leukemic and G0 models. In G0 cells, canonical translation initiation is inhibited; yet we find that inflammatory genes are highly translated, indicating alternative post-transcriptional regulation. Importantly, AU-rich elements (AREs) are significantly enriched in the up-regulated G0 translatome and transcriptome. Mechanistically, we find the stress-responsive p38 MAPK-MK2 signaling pathway stabilizes ARE mRNAs by phosphorylation and inactivation of mRNA decay factor, tristetraprolin (TTP) in G0. This permits expression of ARE-bearing TNF $\alpha$ and DUSP1 that promote chemoresistance. Conversely, inhibition of TTP phophorylation by p38 MAPK inhibitors and non-phosphorylatable TTP mutant decreases ARE mRNAs and sensitizes leukemic cells to chemotherapy. Furthermore, co-inhibiting p38 MAPK and TNFa-prior to or along with chemotherapy—substantially reduced chemoresistance in primary leukemic cells ex vivo and in vivo.

Conclusions: These studies uncover post-transcriptional regulation underlying chemoresistance in leukemia. Our data reveal the p38 MAPK-MK2-TTP axis as a key regulator of expression of ARE bearing mRNAs that promote chemoresistance. By disrupting this pathway, we developed an effective combination therapy against chemosurvival.

Key words: quiescence, chemoresistance, post-transcriptional regulation, AU-rich elements, TTP 


\section{Background}

Quiescent (G0) cells are an assortment of reversibly arrested cells, including dormant stem cells, which are found as a clinically relevant subpopulation in cancers (1-4). Such cells are anti-proliferative, antidifferentiation, and anti-apoptotic, and show distinct properties including resistance to harsh conditions $(1 ; 2 ; 5-10)$. G0 cells show specific gene expression that may underlie their resistance and other properties (1;2;8-10). Analyses from multiple groups revealed some genes up-regulated at the transcriptional level $(1 ; 8 ; 11)$. Altered polyadenylation site selection on mRNAs produces longer $3 \square$ -

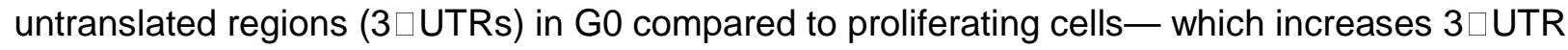
elements that can mediate post-transcriptional gene expression regulation (12). Our previous data demonstrated that translation mechanisms are distinct in G0 leukemic cells, with decreased canonical translation mechanisms and increase in mRNA translation by alternative mechanisms that involve noncanonical translation initiation factors (13) and 3'UTR mediated specific mRNA translation (14). These data suggest that alternate post-transcriptional mechanisms in G0 cancer cells may regulate a distinct translatome to mediate their resistance. Translated genes, post-transcriptional mechanisms involved, and outcomes on cancer persistence remain to be investigated.

We analyzed the translatome and proteome of chemotherapy-surviving G0 cancer cells, focusing on acute monocytic leukemia (AML), to provide comprehensive information that complement and expand previous transcriptome analyses $(1 ; 2 ; 8 ; 11 ; 15 ; 16)$, revealing critical genes that are post-transcriptionally regulated for chemo-survival. G0 can be induced by growth factor-deprivation or serum-starvation and other conditions that isolate dormant cancer stem cells in distinct cell types $(1 ; 6 ; 7)$. Our data demonstrate that serum-starvation induced G0 AML cells are chemoresistant-similar to surviving AML cells, isolated after chemotherapy. Chemoresistant cells isolated via serum-starvation, or as surviving cells post-chemotherapy, show inhibition of canonical translation mechanisms, indicating that noncanonical mechanisms express specific mRNAs when these cells are chemoresistant. Consistently, the translatomes and proteomes of serum-starved G0 and chemo-surviving cells show greater similarity 
than the transcriptome alone. Our data reveal that DNA damage and stress signaling cause posttranscriptional alterations to produce a specialized gene expression program of pro-inflammatory, immune effectors that elicit chemosurvival.

\section{Results}

\section{Serum-starvation or AraC treatment induces a quiescent and chemoresistant state of leukemic} cells

To study clinical resistance in cancer, THP1 human AML cells were used as they show significant resistance to AraC (17) (cytosine arabinoside, Fig. S1A), a standard anti-leukemic chemotherapeutic that targets DNA replication and thus proliferating cells (referred to as $\mathrm{S}_{+}$). Our data and others find that serum-starvation of THP1 (13) and other cell lines $(1 ; 8 ; 11 ; 18)$ induces a transient G0 state with known G0 and cell cycle arrest markers expressed (Fig. 1C-D, S1B-C). Such serum-starvation induced G0 cells (referred to as SS) can be returned to the cell cycle upon serum addition (Fig. 1D), verifying that they are quiescent and transiently arrested, unlike senescence or differentiation that are not easily reversed. We find that serum-starvation induced G0 SS cells show resistance to AraC chemotherapy. Serum-grown S+ cells show a dose-dependent decrease in cell viability with AraC as expected, while SS cells persist, indicating their chemoresistance (Fig. 1E). Chemoresistant cancer cells include cancer stem cells and are a subpopulation that can be isolated from cancers after treatment with chemotherapy (2;6-10) that targets and eliminates S+ cells. We find that AraC-surviving THP1 (referred to as AraCS) cells are transiently arrested, like SS cells (Fig. 1C-D, S1B); both AraCS and SS cells survive chemotherapy (Fig. 1E). AraCS cells recover from their transient arrest upon AraC removal, proliferate (Fig. 1D), affirming the reversible G0 arrest state of chemoresistant cells, similar to SS cells (1;2;6-10). 


\section{GO cells induced by SS or AraC have similar translatomes and proteome features that} recapitulate gene expression profiles of in vivo chemoresistant leukemic and G0 models

To study post-transcriptionally regulated genes in G0, we profiled S+, SS cells and AraCS cells at the proteome, translatome and transcriptome levels using multiplexed quantitative proteomics (14), microarray analysis of heavy polysome-associated mRNAs $(13 ; 14 ; 19)$, and total RNAs respectively (Fig. 1A-B, S1D-F). Notably, we find that AraCS and SS cells show more similar gene expression profiles at the proteome and translatome levels, compared to transcriptome levels (Fig. 1F). These data suggest that although these chemoresistant G0 cells are isolated via two different methods, they exhibit a common set of translatome and proteome, which could underlie their common characteristic of chemoresistance. These data indicate the relevance of examining both the translatome and transcriptome. Time-course translatome analysis revealed that SS G0 cells that were serum-starved for short periods (4 hours and 1 day), are distinct from SS G0 cells that were serum-starved for long periods ( 2 days and 4 days) (Fig. S1E-F). This is consistent with G0 as a continuum of assorted, arrested states (1), with temporal differences in underlying gene expression in early G0 compared to more homogeneity at late G0. SS and AraCS cells provide sufficient material to perform concurrent translatome, proteome and transcriptome profiling, compared to limited cells from in vivo resistance models where only transcriptomes were profiled. To test whether our G0 leukemic cells are relevant models to study chemoresistance and G0, gene expression profiles of AraCS and SS cells were compared to published transcriptome profiles of leukemia stem cells (LSC) from AML (16), dormant leukemic cells (LRC), and minimal residual disease (MRD) from chemotherapy surviving patient samples with acute lymphocytic leukemia (ALL) (15), as well as SS G0 fibroblasts (G0 HFF) (1). Importantly, we find that these published transcriptome signatures for in vivo chemoresistance and G0 models were significantly up-regulated in our SS and AraCS cells (referred to as resistant G0 leukemic cells), compared to S+ cells (Fig. 1G, S1G). These data indicate that our resistant G0 leukemic cells 
are relevant models to study post-transcriptional regulation in chemoresistance as they have similar gene expression profiles to known transcriptional profiles from in vivo chemoresistance models.

\section{Inhibition of canonical translation initiation in resistant G0 leukemic cells}

We find overall protein synthesis is reduced at least 2-fold in AraCS, compared to S+ cells (Fig. 2B, S1D). Mechanistically, both rate-limiting steps in canonical translation initiation: recruitment of initiator tRNA, and mRNA cap recognition to recruit mRNAs to ribosomes are inhibited in G0 leukemic cells (Fig. 2A). Recruitment of initiator tRNA by elF2 can be blocked by elF2a phosphorylation as a stress response (13;20-25). We find that two elF2 kinases, PKR and PERK, are activated and increase elF2 $\alpha$ phosphorylation (Fig. 2C) in G0 leukemic cells to inhibit canonical translation initiation. Consistent with our previous study (14), we observed dephosphorylation of 4E-BP (Fig. 2C) that inhibits cap-dependent translation initiation $(26 ; 27)$. Low mTOR activity is known to reduce translation of terminal oligopyrimidine tract (TOP) mRNAs such as ribosomal protein mRNAs (26;28;29), which is decreased in SS and AraCS cells (Fig. 2D). Decreased canonical translation can enable post-transcriptional regulation of specific genes, as observed previously $(13 ; 14)$ and lead to survival of G0 leukemic cells.

\section{Global translatome analysis shows that inflammatory response genes are selectively translated in resistant $\mathbf{G 0}$ cancer cells}

We measured the number of genes upregulated at the transcriptome, translatome and proteome levels in resistant G0 leukemic cells, compared to S+ cells. A significantly greater number of genes were upregulated in the translatome (580 genes, Table S1) and proteome (716 genes), compared to the transcriptome (318 genes) as shown in Fig. 2E. Importantly, 57\% of upregulated genes were upregulated only in the translatome level (Fig. 2F) but not in the transcriptome, indicating post- 
transcriptional regulation. To investigate the biological function of these differentially expressed genes, gene ontology (GO) analysis was performed. Gene categories up-regulated in G0 translatomes include inflammatory response, immune modulators (receptors, antigen presentation and processing genes), cell adhesion, cell migration, lipid biosynthesis and cholesterol pathway genes (Fig. 2G, S2E). Downregulated genes include RNA processing and ribosome genes (Fig. 2G). To identify translationally upregulated genes, we measured the change in ribosome occupancy $(\mathrm{RO})$ which is the ratio of polysomeassociated mRNA levels to total mRNA levels of each gene (Fig. 2F, Venn diagram, heat map). We find 180 genes are translationally up-regulated above RNA level changes. These genes include antigen processing and presentation genes (30) (HLA-G, HLA-E) and immune receptors (CD47, Fig. 2F-G, S2I) (31-33) that regulate anti-tumor immune response and are associated with leukemic stem cells and resistance $(34 ; 35)$.

We asked if this specific gene expression profile in resistant G0 leukemic cells is conserved in G0 cells of other tumors and normal cells. Therefore, global translatome profiling was conducted in G0 cells from four different cells lines: breast cancer (MCF7), liver cancer (HEP-G2), and osteosarcoma (U2OS) as well as non-cancerous fibroblasts (HFF) (Fig. S2). Their translatome profiles were compared with resistant G0 leukemic cells, using GSEA and DAVID tools (Fig. 2H-I, S2E-F). We find that 580 signature genes of resistant G0 leukemic cells (Table S1) were highly upregulated at the translatome level in G0 cells of these other cell types (Fig. 2H). As expected for these arrested cells, genes related to cell cycle, ribosome biogenesis, and DNA replication were commonly down-regulated (Fig. 2I, S2E). Importantly, inflammatory response genes were commonly up-regulated in cancer G0 cells but not normal G0 fibroblasts and do not significantly overlap with senescence-associated secretory pathway (SASP) (Fig. 2I, S2G) (36;37), indicating a specific role in chemoresistant cancer cells. 


\section{Stabilization of ARE-bearing mRNAs is mediated by phosphorylation of TTP in resistant G0 leukemic cells}

To identify cis-acting elements that mediate post-transcriptional regulation, the untranslated regions (UTRs) of differentially expressed genes were examined. We find that a GC-rich motif was enriched on 5'UTRs of translationally up-regulated genes and an AU-rich motif, on 5'UTRs of down-regulated genes, indicating that mRNAs with structured 5'UTRs are highly translated in G0 cells (Fig. S3A-B).

Importantly, 3'UTR AU-rich elements (AREs) are significantly enriched in the up-regulated translatome as well as transcriptome (Fig $3 \mathrm{~A})$. Furthermore, $30 \%$ of the translatome signature of G0 leukemic cells bear AREs (Table S2), including proinflammatory cytokines such as TNFa and chemokines (Fig. 3B-C). AREs are important post-transcriptional regulatory elements that mediate rapid degradation and repression of mRNAs (38). To understand how ARE mRNAs are highly expressed in G0 cells, we assessed the expression level of RNA-binding proteins. As expected, ARE-binding proteins known to cause mRNA decay or translation repression $(39 ; 40)$ are significantly reduced in G0 cells (Fig. S3F). Additionally, the exosome and proteasome complexes that are implicated in ARE mRNA decay (41) (42) are reduced (Fig. S3C-E). However, a key ARE mRNA decay and translation repression factor, Tristetraprolin (TTP) was surprisingly increased in AraCS from multiple AML cell lines (Fig. 3D-E). However, we find that TTP is phosphorylated in SS and AraCS cells (Fig. 3E, right). TTP phosphorylation is established to increase its levels (43), and block its ability to destabilize ARE mRNAs, thus enabling ARE mRNA translation upon LPS treatment $(44 ; 45)$. To test whether phosphorylation of TTP was required for the increased expression of ARE mRNAs in G0 leukemic cells, we generated non-phosphorylatable mutant TTP with key phosphorylation sites (Ser 52,178$)$ replaced by alanine (TTP-AA). TTP-AA has been shown to dominantly maintain ARE mRNA decay activity and reduce pro-inflammatory cytokines like TNFa in immune cells (43-45). Expression of myc-tagged TTPAA significantly reduced TNFa mRNA in both THP1 and K562 AraCS cells (Fig. 3F), overturning the decay inactivity of endogenous phospho-TTP. To determine the effect of TTP phosphorylation on the 
stability of ARE mRNAs, we measured the half-life of TNFa mRNA. Expression of TTP-AA mutant more significantly reduced the half-life of TNFa mRNA than TTP wild-type expressed in AraC-treated TTPdeficient macrophages ${ }^{48}$ (Fig 3G). Furthermore, immunoprecipitation demonstrated that TTP-AA was associated with TNFa mRNA in AraCS cells (Fig 3H). These data indicate that inactivation of ARE mRNA decay by TTP phosphorylation $(43 ; 45 ; 46)$ is a key regulator of expression of a pro-inflammatory gene, TNFa, in chemoresistant G0 cells. These results are consistent with our findings of increased levels and translation of ARE bearing mRNAs due to decreased ARE mRNA decay activity in G0 cells (Fig. 3A-C, S3C-F).

\section{The p38 MAPK-MK2 pathway phosphorylates TTP to promote expression of ARE-bearing}

\section{mRNAs in resistant G0 leukemic cells}

To investigate how TTP is phosphorylated in resistant G0 leukemic cells, we examined key signaling molecules involved in DNA-damage response (DDR) (Fig. 4A) that is induced by chemotherapies like AraC (47-50). As expected, AraC treatment induced rapid phosphorylation and activation of ATM (Fig. 4B). Importantly, we find that these conditions lead to phosphorylation and activation of p38 MAPK and its downstream effector, MAPKAPK2 (MK2) $(51 ; 52)$ (Fig. 4B). MK2 has been shown to phosphorylate TTP in macrophages treated with lipopolysaccharide (LPS) $(43 ; 45 ; 46)$. To examine whether the p38 MAPK-MK2 pathway phosphorylates TTP in resistant G0 leukemic cells, two different inhibitors of p38 MAPK were tested. Treatment with p38 MAPK $\alpha / \beta$ inhibitor, LY2228820 (LY) $(52 ; 53)$, or a pan-p38 MAPK inhibitor that targets all isoforms, BIRB796 (BIRB) (54), blocked phosphorylation of MK2 and prevented MK2-mediated TTP phosphorylation and reduces TNFa in AraCS cells (Fig. 4C). These results suggest that p38 MAPK-MK2 phosphorylates TTP, resulting in enhanced expression of ARE mRNAs such as TNFa upon AraC treatment (Fig. 4A). To test if the p38 MAPK-MK2-TTP pathway regulates TNFa expression via its ARE, a firefly luciferase reporter bearing the $3^{\prime}$ UTR ARE of TNFa, 
and as control, Renilla luciferase, were co-transfected. Luciferase activity of the ARE reporter increased by 2-fold in AraCS cells compared to S+ cells but not when p38 MAPK was inhibited (Fig. 4D). These data suggest that the p38 MAPK-MK2-TTP axis up-regulates expression of specific genes via AREs in G0 leukemic cells.

\section{Phosphorylation of TTP induced by p38 MAPK-MK2 promotes chemoresistance}

We noted that the p38 MAPK-MK2 pathway was rapidly activated to phosphorylate TTP within one day of SS or AraC treatment (Fig. 4B, S4A-B). To test the effect of inhibition of TTP phosphorylation on chemoresistance, p38 MAPK was inhibited before (or along with) as well as after treatment with AraCand then chemosurvival was measured using multiple assays, including cell death and two cell viability assays (Fig. 4E-G). Inhibition of p38 MAPK with BIRB or LY, one day after AraC treatment, when TTP was already phosphorylated, did not show any significant reduction in survival of AraC-resistant cells (Fig. 4F-G). Conversely, inhibition of p38 MAPK at earlier time points prior to AraC treatment, when TTP was not phosphorylated, increased apoptosis and reduced survival of AraC-resistant cells (Fig. 4FG). As a control, p38 MAPK inhibition alone does not affect viability of S+ cells that are not treated with AraC (Fig. 4F-G). These results suggest that p38 MAPK is rapidly activated upon AraC treatment to turn on downstream survival pathways such as phosphorylation of TTP. Thus, to inhibit phosphorylation of TTP and hence overcome AraC resistance effectively, p38 MAPK needs to be targeted at early time points.

To confirm that phosphorylation of TTP induces chemoresistance, we over-expressed TTP mutant (TTP-AA) that cannot be phosphorylated by p38 MAPK-MK2, followed by AraC treatment. Importantly, we find that TTP-AA mutant expression reduces survival of AraC-resistant cells in THP1 and K562 leukemic cell lines (Fig. 4H). Furthermore, TTP-AA mutant, expressed in TTP-knockout macrophages, 
induced apoptosis of AraC-surviving cells more significantly, compared to TTP wild-type (Fig. 4I). Consistently, in multiple AML cell lines, early inhibition of p38 MAPK showed dramatically reduced chemosurvival but not in non-cancerous CD34+ cells (Fig. 4J). When treated with p38 MAPK inhibitor alone, viability of $\mathrm{S}+$ cells in multiple AML cell lines remained unchanged, indicating the synergism of AraC and p38 MAPK inhibitors (Fig. 4J). Interestingly, p38 MAPK inhibition eliminated resistant cells more significantly at increasing concentrations of AraC (Fig. 4K). This indicates that treatment with high concentrations of AraC would increase the number of cells induced into the resistant G0 state with strong phosphorylation of p38 MAPK-MK2-TTP. Conversely, even low concentrations of BIRB were sufficient to reduce chemoresistance (Fig. S4C). Unlike in solid tumors, where activation of p38 MAPKMK2 induces resistance by arresting the cell cycle $(38 ; 51 ; 52)$, p38 MAPK inhibition did not affect the cell cycle in AML cells (Fig. S4D). These data uncover rapid activation of a p38 MAPK-MK2 pathway that enables chemosurvival of G0 leukemic cells via inhibition of TTP activity.

\section{TNFa, induced by phosphorylation of TTP, promotes chemoresistance}

We demonstrated that TTP regulates the stability of ARE mRNAs such as TNF $\alpha$ in AraCS cells (Fig. 3G). Furthermore, inactivation of TTP allowed elevated TNF $\alpha$ translatome and protein levels in resistant G0 leukemic cells (Fig 5B-C). To assess the effect of TNFa on chemoresistance, we altered TNFa levels genetically and phamacologically in G0 cells (Fig. 5A). Induction of TNF $\alpha$ depletion prior to AraC effectively reduced AraC resistance, compared to depleting TNFa after AraC treatment, while no effect was observed with TNF $\alpha$ depletion alone without AraC (Fig. 5D). In contrast, addition of recombinant TNF $\alpha$ enhanced survival of AraCS cells (Fig. 5D). TNF $\alpha$-mediated chemoresistance is not due to arrested cell cycle as TNF $\alpha$ treatment without subsequent AraC does not alter the cell cycle 
(Fig. S5C). These data suggest that phosphorylation of TTP and subsequent expression of TNFa, which are induced by p38 MAPK-MK2, are responsible for survival of G0 leukemic cells.

TNF $\alpha$ can also be inhibited pharmacologically with the drug pirfenidone (PFD) that can block TNF $\alpha$ translation in RAW264.7 cells and is used to treat idiopathic pulmonary fibrosis $(52 ; 55 ; 56)$. In G0 leukemic cells, PFD reduced TNF $\alpha$ translatome and protein levels but not mRNA levels (Fig. 5E, S5F). PFD treatment at least 18 hours prior to or along with AraC or SS significantly reduced viability of G0 leukemic cells but failed to reduce resistance when added after AraC treatment (Fig. 5F, S5D). As observed with p38 MAPK-MK2 activation (Fig. 4A-B), TNF $\alpha$ translatome level also is rapidly and dramatically increased upon SS treatment (Fig. 5B). These data indicate that activation of TNF $\alpha$ is an early event in G0 induction, which leads to resistance, and needs to be inhibited early to preclude downstream survival regulators. PFD treatment alone does not affect the viability of untreated S+ cells, indicating that the cytotoxic effect of PFD is specific to G0 leukemic cells (Fig. 5F). PFD treatment reduced chemotherapy survival in multiple AML cell lines (Fig. 5G). Similar results were observed in MCF7 cells, where PFD reduced doxorubicin resistance (Fig. S5H).

TNF $\alpha$ activates the NFKB pathway that increases anti-apoptotic gene expression to promote cell survival (57-59). Our observation of early activation of p38 MAPK-MK2 (Fig. 4A-B) suggested that TNFa could be rapidly up-regulated upon G0 induction. Time-course translatome analysis affirmed that TNF $\alpha$ is highly increased (16-fold) at the earliest time point of $4 \mathrm{~h}$ after serum-starvation or AraC treatment (Fig. 5B) along with its receptors, leading to rapid elevation of downstream NFאB target genes including anti-apoptotic BCL family members (59-61) (Fig. 5B, S5A-B). Similar to our observations with TNF $\alpha$ inhibitor PFD, NFאB inhibitor, BAY11-7082(62) prior to or along with AraC or SS decreases survival of G0 cells, while treatment after AraC or SS had no effect (Fig. 5H). These data suggest that the TNF $\alpha-N F \kappa B$ inflammatory pathway is upregulated as an early survival pathway in G0 cells. 


\section{TTP regulates a pro-apoptotic JNK pathway via targeting DUSP1}

We asked what other ARE mRNAs are targeted by TTP and affect cell survival. DUSP1 mRNA contains AREs in its 3' UTR. TTP has been shown to target DUSP1 mRNA for degradation upon LPS treatment of macrophages or dendritic cells $(44 ; 45 ; 63)$. To determine if TTP phosphorylation regulates DUSP1 in AraCS, we expressed TTP-AA mutant that is not phosphorylated in BMDM cells that lack TTP (Fig. 6A). Immunoprecipitation showed that TTP-AA associated with DUSP1 mRNA in AraCS cells (Fig. 6B). Expression of TTP-AA mutant more significantly reduced DUSP1 mRNA and protein levels compared to cells expressing TTP wild-type (Fig. 6C-D). Furthermore, inhibition of phosphorylation of TTP by p38 MAPK inhibitor decreased DUSP1 protein level (Fig. 6E). DUSP1 is a MAPK phosphatase which dephosphorylates JNK (64). In AraCS cells, DUSP1 protein level is negatively correlated with phosphorylated JNK (Fig. 6C, 6E), consistent with DUSP1-mediated suppression of JNK(64). To determine the effect of JNK on survival of leukemic cells, JNK inhibitor, JNK-IN-8 was used (Fig. 6A). Importantly, JNK inhibition reversed apoptosis of leukemic cells treated with AraC, LY and PFD, but did not affect the viability of untreated cells (Fig. 6F), indicating that inhibition of JNK pathway contributes to chemoresistance. Together, these results suggest that TTP-DUSP1 axis promotes chemoresistance via suppressing JNK-mediated apoptosis (Fig. 6A).

\section{Co-inhibition of p38 MAPK and TNFa sensitizes resistant leukemic cells to AraC treatment}

Although chemoresistant cells are sensitive to individual inhibition of either TNFa or p38 MAPK by PFD or LY respectively, a substantial proportion of cells still survived (Fig. 4F, 5G). Therefore, we asked if coinhibition of p38 MAPK and TNFa with LY and PFD respectively, could eliminate the remaining resistant cells. We find that individual treatment with either of LY or PFD prior to or along with AraC, reduces approximately $50 \%$ of surviving leukemic cells (Fig. 7B). Importantly, the combination of PFD and

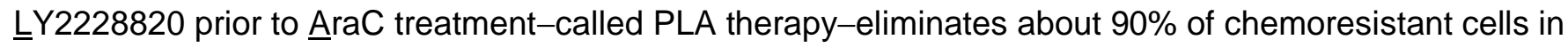


multiple AML cell lines (Fig. 7A-C). Furthermore, PLA therapy decreased colony formation capacity of leukemic cells on methylcellulose by 10-fold, compared to AraC-treatment alone (Fig. 7D). These data indicate a severe loss of stem cell capacity of leukemic cells treated with PLA therapy. In contrast, in the absence of AraC treatment, the combination of PFD and LY2228820 did not affect cell viability, apoptosis and colony formation capacity, indicating the synergistic effect between AraC and anti-inflammatory drugs (Fig. 7B-D). Despite the fact that stromal niche cells have been shown to protect leukemic cells from chemotherapy (65), we find that AML cells co-cultured with stromal cells remained sensitive to PLA therapy (Fig. S5E). We investigated the molecular mechanism by which PLA therapy enhanced chemosensitivity. We find that LY treatment destabilizes TNFa mRNAs by TTP dephosphorylation (43)

(Fig. 3G, 4C), while PFD suppresses translation of TNFa mRNA (56) (Fig. 5E). Therefore, in PLA therapy, TNFa remains more effectively blocked, compared to individual drug treatments (Fig. 6E). Furthermore, a pro-apoptotic JNK pathway was more significantly activated in cells treated with PLA therapy than singledrug treatments (Fig. 6E). Together, these results suggest that PLA therapy reduces TNFa and promotes a pro-apoptotic JNK pathway, leading to apoptosis of chemoresistant cells.

\section{PLA therapy reduces chemoresistance in primary AML cells ex vivo and in vivo}

To test the anti-leukemic activity of PLA therapy in primary AML (66), primary cells from AML patients as well as two murine AML models driven by Hoxa9/Meis1 or MLL/AF9 (SI Methods), were used. When either p38 MAPK or TNFa was inhibited prior to AraC treatment, moderate apoptosis of chemoresistant cells was observed in primary AML cells (Fig. 8A-B). Importantly, co-inhibition of p38 MAPK and TNFa by PLA therapy (pre-treatment before AraC) significantly reduced AraC resistance in fourteen out of fifteen AML patient samples as well as in primary cells from two AML mouse models ex vivo (Fig. 8AB). In contrast, the viability of normal CD34+ cells from healthy donors was not affected by treatment with LY or PFD (Fig. 4J, 8A-B), consistent with clinical studies that have shown that PFD and LY have 
acceptable safety and tolerance $(53 ; 55)$. To further investigate the therapeutic potential of PLA therapy in vivo, human AML cells expressing luciferase (MOLM13-Luc, SI Methods) were intravenously or subcutaneously injected into NSG mice. After confirmation of engraftment by measuring tumor volume or bioluminescent imaging (BLI), the mice were treated with PLA therapy or AraC for two weeks.

Consistent with ex vivo results (Fig. 7B), PLA therapy significantly decreased the leukemic burden and tumor volume by 6-fold, compared to AraC treatment alone (Fig. 8C-D). Next, primary Hoxa9/Meis1 or MLL/AF9 leukemia cells were generated as described previously (67), and transplanted to second recipient mice. These mice were treated with PLA therapy or AraC. Consistently, BLI shows that PLA therapy eliminated $78 \%$ or $96 \%$ of chemoresistant cells in a dosage-dependent manner (Fig. $8 \mathrm{E}-\mathrm{F}$ ) and extended mice survival (Fig. $8 \mathrm{H}$ and S5G). In the absence of AraC treatment, the combination of PFD and LY2228820 did not affect leukemic burden, suggesting that cytotoxic effects of this combination are limited to AraC-resistant cells, rather than proliferating cells (Fig. 8G). Together, these results suggest PLA therapy has potential for improving AraC-mediated apoptosis in AML.

\section{Discussion}

G0 cells are a transiently arrested, clinically relevant subpopulation in cancers $(1 ; 2 ; 5-10)$. Our previous data and others, revealed altered gene expression mechanisms in G0 leukemic cells, at the posttranscriptional $(8 ; 12)$ and translational levels $(13 ; 14 ; 18)$. This would lead to a distinct gene expression profile to enable G0 cell survival in harsh conditions. G0 cells are resistant to stress conditions like serum-starvation, with transient inhibition of apoptosis, and proliferation $(1 ; 11 ; 18)$. Importantly, we find that serum-starved leukemic SS G0 cells exhibit chemoresistance (Fig. 1E); consistently, true chemosurviving AraCS cells are transiently arrested and chemoresistant (Fig. 1D-E, S1B-C). In accord, we find that SS cells are similar in translatome and proteome to AraCS cells (Fig. 1F), indicating that consistent with their common features of G0 arrest and chemosurvival, they show similar post- 
transcription gene expression. Published transcriptional signatures of in vivo chemoresistance leukemic models $(1 ; 2 ; 8 ; 11 ; 15 ; 16)$, are also highly expressed in SS and AraCS cells (Fig. 1G, S1G). Thus, the common G0 resistance gene expression profile observed in AraCS and SS G0 cells likely comprises genes that control survival and resistance. These data revealed that in addition to known transcriptional profiles, altered post-transcriptional mechanisms in G0 resistant cells contribute to their unique gene expression profile that underlies their chemoresistance.

Our findings reveal the importance of DNA damage and stress signaling that can initiate a proinflammatory response that causes survival (Fig. 4). Differential genomic instability in cancers would lead to subpopulations within a tumor with disparate DDR and stress signaling (47-49) that we find, enables their chemotherapy survival via pro-inflammatory cytokines. Cytokines upregulated in SS and AraCS cells include some SASP factors but also other unique cytokines (36;37) (Fig. S2G). This is consistent with similarities and differences between G0 and senescence (1): both show low mTOR activity but G0 shows reversible arrest, stem cell markers, low p53 and lack of senescence markers (Fig. 2C-D, 2I, S1G) (14)—unlike senescence(18). These data indicate that a quiescence- and resistance-specific set of pro-inflammatory and signaling genes are expressed in these resistant cells (Fig. 2G). These include inflammatory cytokine, TNFa that promotes downstream NFкB activated prosurvival target genes (57-59) including BCL family members of anti-apoptotic genes (59-61) (Fig. 5A-C, S5A-B). Treatment with anti-inflammatory reagents after chemotherapy is not very effective as the downstream survival effectors have already been induced; thus, targeting their upstream cytokine regulators would not be effective at this later time (Fig. 4F-G, 5F-H, S5D). Therefore, treatment with reagents that block these resistance pathways prior to (and continued with) or along with chemotherapy, enables the most effective reduction of resistance, as they prevent further enrichment of such resistant cells by blocking induction of pro-survival signaling. 
Increasing AraC, a nucleotide analog that inhibits replication (17), would activate DDR and downstream p38 MAPK signaling (47-49) and should lead to more cells expressing this inflammatory pathway that enables resistance. Consistently, increased AraC treatment leads to more cells in the inflammatory phase that can be targeted by LY to curb resistance (Fig. 4K). Non-cancerous cells do not show this pathway (Fig. 2l) and are not affected by inhibitors (Fig. 4J, 8A). These data suggest that certain chemotherapies and stresses like serum-starvation induce stress signaling (Fig. 4A-C) and enrich for resistant G0 cells-in addition to pre-existing subpopulations with genomic instability that trigger DDR and stress (47-49). Importantly, this resistance mechanism can be blocked, not only in different AML cell lines (Fig. 4J, 5G, 7B) but also in vivo (Fig. 8C-G) and in multiple patient-derived primary AML—without affecting normal cells (Fig. 8A)—supporting their potential applicability as a therapeutic against chemoresistance in AML.

We find key signaling pathways induced by AraCS and SS treatments, which alter post-transcriptional and translational gene expression to enable resistance. These include: 1. DNA damage ATM (47-49) and stress activated p38 MAPK signaling that in turn promotes MK2 $(51 ; 52)$ that post-transcriptionally upregulates ARE bearing mRNAs $(43 ; 45 ; 46)$. The expressed mRNAs include ARE-bearing proinflammatory cytokine TNFa $(57 ; 58)$ that activates downstream anti-apoptosis signals (Fig. 4A-D, 5A-C, S5A-B) (59-61), and ARE-bearing signaling regulator DUSP1(63;64)that blocks JNK-mediated apoptosis (Fig. 6), to promote resistance. 2. ATM-mediated suppression of mTOR activity $(47 ; 48)$ that inhibits canonical translation initiation via 4EBP dephosphorylation (Fig. 2A-C); this results in specific translation of pro-inflammatory cytokines(14) (Fig. 3A-C) and immune modulators (30) (HLA-G, CD47, Fig. 2F, S2I) (31-33) that regulate anti-tumor immune response and resistance $(34 ; 35)$. 3. UPR stress signaling, induced downstream of p38 MAPK (68) and DNA damage (69;70), also inhibits canonical translation via PERK phosphorylation of elF2 $\alpha$ and enables non-canonical specific mRNA translation (Fig. 2A-D, S2H)(69;70). Blocking the p38 MAPK $\alpha / \beta$ pathway with LY $(52 ; 53)$, in combination with the 
anti-inflammatory PFD $(52 ; 55 ; 56)$ that precludes downstream TNF $\alpha$ expression $(55 ; 56)$ (Fig. 5E)-prior to (and continued with) AraC chemotherapy-lead to effective loss of chemoresistance in multiple AML cell lines (Fig. 7B), in tumors in vivo in AML mouse models (Fig. 8C-G), and in patient samples (Fig. 8A), validating their ability to reduce resistance and tumors in vitro and in vivo. LY destabilizes TNFa mRNA by TTP dephosphorylation (Fig. 4C) (43), while PFD suppresses TNFa selectively at the translation level (56) (Fig. S5F) and thus enables PLA combination therapy to more effectively curb resistance than the individual drugs (Fig. 7B, 8B). Apart from its effect on TNF $\alpha$ translation, PFD blocks inflammation regulator $(71 ; 72)$ p38 MAPKy that can be increased upon p38MAPK $\alpha / \beta$ inhibition, preventing feedback reactivation of inflammation, and enabling PLA combination therapy to remain more efficacious than the individual drugs. Therefore, the combination of PFD and LY suppresses the inflammatory and stress response more effectively in vitro and in vivo (Fig. 7-8). Upon inhibition of p38 MAPK, in addition to reduction of TNFa and its downstream anti-apoptotic signals, we find the ARE bearing DUSP1 is reduced, leading to activation(63;64)of the JNK pathway(73) to promote apoptosis (Fig. 6E-F). These data indicate that blocking pro-inflammatory effectors-that are induced by chemotherapy mediated DNA damage and stress signaling-leads to increased chemosensitivity and decreased resistant cell survival.

Our findings revealed that these pro-inflammatory and signaling genes upregulated in G0, have AREs and other UTR sequences that regulate mRNA levels and translation (Fig. 3A-C, S3A). The ATM-p38 MAPK-MK2 axis stabilizes these ARE bearing pro-inflammatory cytokine and signaling mRNAs by phosphorylating ARE binding mRNA decay factor, TTP to prevent its mRNA decay activity on proinflammatory cytokine TNFa (Fig. 3D-H, 4C-D) and signaling regulator, DUSP1 (Fig. 6A-D). In support, overexpression of TTP-AA — that cannot be phosphorylated and is a dominant active form that restores ARE mRNA decay (43-45)—decreases TNFa and DUSP1 expression (Fig. 3F-G, 6A-D), and thereby reduces chemoresistance (Fig. 4H-I, 6E-F). This is consistent with previous studies on AREs in cancers 
$(14 ; 38 ; 43 ; 74-77)$. These data suggest that phospho-TTP level or TTP activity is an important regulator of inflammatory response mediated chemoresistance, which can be harnessed as a marker and target against AML resistance. Consistently, published in vivo leukemia resistance models show increased expression of TTP and ARE bearing genes (15;78), similar to our studies (Fig. 3A-E). Our studies on TTP and ARE regulated immune and signaling modulators that promote chemoresistance, are consistent with recent findings of TTP regulation of PDL1 to mediate immunoresistance in solid tumors(79). Importantly, inhibition of these pathways curtails chemoresistance and tumor survival in vivo in primary AML patients and tumor models (Fig. 8). Together, these pathways that are upregulated in resistant cells (Fig. 4A, 5A) via chemotherapy and stress induced signaling-decrease canonical translation and permits non-canonical post-transcriptional regulation of specific genes (Fig. S6)- to promote chemosurvival of G0 cancer cells.

\section{Conclusions}

Our studies reveal that G0 leukemic cells are chemoresistant, indicating their clinical importance in cancer persistence. We find a specific proteomic and translation profile that is induced commonly between G0 cells and chemosurviving leukemic cells. We uncovered critical genes that are specifically upregulated post-transcriptionally and translationally for cell survival in these conditions by key survival signaling pathways. These studies reveal the significance of post-transcriptional and translational regulation of immune and signaling modulators in chemoresistance. Our data enabled the development of a new combination therapy to effectively reduce resistance in cancer cell lines, in tumors in vivo, and in patient tumor samples, without affecting normal cells. 


\section{Methods}

Overview, aim, design, and setting Therapeutic targeting of minimal residual disease or chemoresistant, leukemic stem cells in leukemias, particularly acute myeloid leukemia, has been ineffective thus far and refractory leukemia is fatal. The mechanisms of translation and posttranscriptional control, and the critical translation profile that control the ultimate, specific protein profile, and thereby-survival of such clinically resistant cells—are largely undiscovered. Therefore, we globally analyzed gene expression at every level—RNA levels, translatome and proteome-in chemotherapysurviving G0 cancer cells in acute monocytic leukemia and other cancers, the specialized posttranscriptional and translational mechanistic changes, their key signaling regulatory pathways, as well as developed a new, resistance-gene expression targeting therapy to understand and reduce chemoresistance.

Detailed description of characteristics, materials used, and methods including cell culture, patient samples, tumor models, profiling, plasmids, cell viability assays, flow cytometry, protein analysis, drugs, and motif analysis are described in detail in Supplemental Information.

Statistical analyses are described in Supplemental Information.

\section{Declarations}

\section{Ethics approval and consent to participate}

Statement on Human Data All human samples (de-identified) were handled in accordance with IRB protocols to SV (2015P000998/MGH), approved by the Partners Human Research Committee Institutional Review Board /MGH IRB, to DAS, and to Tim Graubert \& J. L-S (DF/HCC 13-583), approved by DF/HCC Office for Human Research Studies. Details in Supplemental Information. 
Statement on animal data nod-scid-gamma (NSG), C57Black/6 mice, 10-12 weeks, are obtained from MGH Cox-7 Gnotobiotic animal facility of the AAALAC-accredited Center for Comparative Medicine and Services at MGH. These facilities are supervised by veterinarians in the Center for Comparative Medicine and the MGH Subcommittee for Animal Research (SRAC) and maintained according to the protocol approved by SRAC, and provide services for breeding, regular health checks, histopathology and macropathology.

2. Consent for publication All samples are de-identified, described under the above IRB protocols.

3. Availability of material and data Raw datasets will be submitted to GEO public repository at final submission. All datasets and material will be made available publicly on publication and on request.

4. Statement on competing interest The authors have declared that they have no competing interests.

5. Funding statement The study is funded by Cancer Research Institute, D. \& M-E Ryder, Leukemia \& Lymphoma Society, Smith Family Foundation, GM100202 grants to SV \& CA185086. SL was funded by Fund for Medical Discovery fellowship \& by a postdoctoral fellowship from the Basic Science Research Program through the National Research Foundation of Korea (2015017218). DAS is funded by $\mathrm{NCI}$ CA115772. DAH is funded by Howard Hughes Medical Institute \& 2R01 CA129933.

6. Author Contributions SL conducted the research and bioinformatic analysis; SST, SIAB \& DL contributed data; SL, YK, DM, BTN, ID-G, DTM, M-KC, DS, SM \& DAH provided in vivo models \& in vivo data; MAM, RR \& RG did immune data; CT \& MD-M provided TTP reagents \& stable knockdown cells, AL, NJH, \& ML provided mRNA folding energies \& patient gene signatures; MB \& WH conducted proteomics; DAS \& JL-S provided patient samples; SV supervised the project \& wrote the manuscript. 
7. Acknowledgments We thank Partners Healthcare Center for Personalized Genetic Medicine \&

BUMC facilities for microarray data; N. Kedersha, S. Lyons \& P. Anderson for plasmids \& antibody; T.

Graubert for patient samples; D. Bloch, S. Wu, A. Naar, M. Gaestel, M. Guzman, N. Bardeesy, D.

Scadden, S. Ramaswamy, \& C. Benes for reagents.

\section{List of Supplemental files:}

\section{Supplemental Information}

Methods (related to main text, main figures 1-8 \& supplemental figures S1-S6)

Supplemental References (related to supplemental information, methods section)

Supplemental Figure legends S1-S6, related to main figures 1-8

\section{Supplemental Figures S1-S6 (related to main figures 1-8)}

3. Supplemental Tables 1-2 (related to main figures 1-8 \& supplemental Figures S1-S6)

\section{Reference List}

(1) Coller HA, Sang L, Roberts JM. A new description of cellular quiescence. PLoS Biol 2006 Mar;4(3):e83.

(2) Ng SW, Mitchell A, Kennedy JA, Chen WC, McLeod J, Ibrahimova N, et al. A 17-gene stemness score for rapid determination of risk in acute leukaemia. Nature 2016 Dec 15;540(7633):433-7.

(3) Meacham CE, Morrison SJ. Tumor heterogeneity and cancer cell plasticity. Nature 2013 Sep 19;501(7467):328-37.

(4) Crews $\mathrm{LA}$, Jamieson $\mathrm{CH}$. Selective elimination of leukemia stem cells: hitting a moving target. Cancer Lett 2013 Sep 10;338(1):15-22.

(5) Bhola PD, Mar BG, Lindsley RC, Ryan JA, Hogdal LJ, Vo TT, et al. Functionally identifiable apoptosis-insensitive subpopulations determine chemoresistance in acute myeloid leukemia. $\mathrm{J}$ Clin Invest 2016 Sep 1;126(10):3827-36. 
(6) Tavaluc RT, Hart LS, Dicker DT, El-Deiry WS. Effects of low confluency, serum starvation and hypoxia on the side population of cancer cell lines. Cell Cycle 2007 Oct 15;6(20):2554-62.

(7) Gupta PB, Onder TT, Jiang G, Tao K, Kuperwasser C, Weinberg RA, et al. Identification of selective inhibitors of cancer stem cells by high-throughput screening. Cell 2009 Aug $21 ; 138(4): 645-59$.

(8) Salony, Sole X, Alves CP, Dey-Guha I, Ritsma L, Boukhali M, et al. AKT Inhibition Promotes Nonautonomous Cancer Cell Survival. Mol Cancer Ther 2016 Jan;15(1):142-53.

(9) Li L, Bhatia R. Stem cell quiescence. Clin Cancer Res 2011 Aug 1;17(15):4936-41.

(10) Giles FJ, DeAngelo DJ, Baccarani M, Deininger M, Guilhot F, Hughes T, et al. Optimizing outcomes for patients with advanced disease in chronic myelogenous leukemia. Semin Oncol 2008 Feb;35(1 Suppl 1):S1-17.

(11) Liu H, Adler AS, Segal E, Chang HY. A Transcriptional Program Mediating Entry into Cellular Quiescence. PLoS Genet 2007 Jun 8;3(6):e91.

(12) Sandberg R, Neilson JR, Sarma A, Sharp PA, Burge CB. Proliferating cells express mRNAs with shortened 3' untranslated regions and fewer microRNA target sites. Science 2008 Jun 20;320(5883):1643-7.

(13) Lee S, Truesdell SS, Bukhari SI, Lee JH, Letonqueze O, Vasudevan S. Upregulation of elF5B controls cell-cycle arrest and specific developmental stages. Proc Natl Acad Sci U S A 2014 Oct 14;111(41):E4315-E4322.

(14) Bukhari SI, Truesdell SS, Lee S, Kollu S, Classon A, Boukhali M, et al. A Specialized Mechanism of Translation Mediated by FXR1a-Associated MicroRNP in Cellular Quiescence. Mol Cell 2016 Mar 3;61(5):760-73.

(15) Ebinger S, Ozdemir EZ, Ziegenhain C, Tiedt S, Castro AC, Grunert M, et al. Characterization of Rare, Dormant, and Therapy-Resistant Cells in Acute Lymphoblastic Leukemia. Cancer Cell 2016 Dec 12;30(6):849-62.

(16) Saito Y, Kitamura H, Hijikata A, Tomizawa-Murasawa M, Tanaka S, Takagi S, et al. Identification of therapeutic targets for quiescent, chemotherapy-resistant human leukemia stem cells. Sci Transl Med 2010 Feb 3;2(17):17ra9.

(17) Forbes SA, Beare D, Gunasekaran P, Leung K, Bindal N, Boutselakis H, et al. COSMIC: exploring the world's knowledge of somatic mutations in human cancer. Nucleic Acids Res 2015 Jan;43(Database issue):D805-D811.

(18) Loayza-Puch F, Drost J, Rooijers K, Lopes R, Elkon R, Agami R. p53 induces transcriptional and translational programs to suppress cell proliferation and growth. Genome Biol $2013 \mathrm{Apr}$ $17 ; 14(4):$ R32.

(19) Hsieh AC, Liu Y, Edlind MP, Ingolia NT, Janes MR, Sher A, et al. The translational landscape of mTOR signalling steers cancer initiation and metastasis. Nature 2012 Feb 22;485(7396):55-61. 
(20) Sendoel A, Dunn JG, Rodriguez EH, Naik S, Gomez NC, Hurwitz B, et al. Translation from unconventional 5' start sites drives tumour initiation. Nature 2017 Jan 26;541(7638):494-9.

(21) Holcik M, Sonenberg N. Translational control in stress and apoptosis. Nat Rev Mol Cell Biol 2005 Apr;6(4):318-27.

(22) Zeenko VV, Wang C, Majumder M, Komar AA, Snider MD, Merrick WC, et al. An efficient in vitro translation system from mammalian cells lacking the translational inhibition caused by elF2 phosphorylation. RNA 2008 Mar;14(3):593-602.

(23) Lorsch JR, Dever TE. Molecular view of $43 \mathrm{~S}$ complex formation and start site selection in eukaryotic translation initiation. J Biol Chem 2010 Jul 9;285(28):21203-7.

(24) Ron D, Walter P. Signal integration in the endoplasmic reticulum unfolded protein response. Nat Rev Mol Cell Biol 2007 Jul;8(7):519-29.

(25) Zismanov V, Chichkov V, Colangelo V, Jamet S, Wang S, Syme A, et al. Phosphorylation of elF2alpha; Is a Translational Control Mechanism Regulating Muscle Stem Cell Quiescence and Self-Renewal. Cell Stem Cell 2016 Jan 7;18(1):79-90.

(26) Thoreen CC, Chantranupong L, Keys HR, Wang T, Gray NS, Sabatini DM. A unifying model for mTORC1-mediated regulation of mRNA translation. Nature 2012 May 3;485(7396):109-13.

(27) Culjkovic B, Topisirovic I, Borden KL. Controlling gene expression through RNA regulons: the role of the eukaryotic translation initiation factor elF4E. Cell Cycle 2007 Jan 1;6(1):65-9.

(28) Han K, Jaimovich A, Dey G, Ruggero D, Meyuhas O, Sonenberg N, et al. Parallel measurement of dynamic changes in translation rates in single cells. Nat Methods 2014 Jan;11(1):86-93.

(29) Damgaard CK, Lykke-Andersen J. Translational coregulation of 5G TOP mRNAs by TIA-1 and TIAR. Genes Dev 2011 Oct 1;25(19):2057-68.

(30) Shukla SA, Rooney MS, Rajasagi M, Tiao G, Dixon PM, Lawrence MS, et al. Comprehensive analysis of cancer-associated somatic mutations in class I HLA genes. Nat Biotechnol 2015 Nov;33(11):1152-8.

(31) Sosale NG, Spinler KR, Alvey C, Discher DE. Macrophage engulfment of a cell or nanoparticle is regulated by unavoidable opsonization, a species-specific 'Marker of Self' CD47, and target physical properties. Curr Opin Immunol 2015 Aug;35:107-12. doi: 10.1016/j.coi.2015.06.013. Epub;\%2015 Jul 13.:107-12.

(32) Soto-Pantoja DR, Kaur S, Roberts DD. CD47 signaling pathways controlling cellular differentiation and responses to stress. Crit Rev Biochem Mol Biol 2015;50(3):212-30.

(33) Zhang H, Lu H, Xiang L, Bullen JW, Zhang C, Samanta D, et al. HIF-1 regulates CD47 expression in breast cancer cells to promote evasion of phagocytosis and maintenance of cancer stem cells. Proc Natl Acad Sci U S A 2015 Nov 10;112(45):E6215-E6223. 
(34) de Kruijf EM, Sajet A, van Nes JG, Natanov R, Putter H, Smit VT, et al. HLA-E and HLA-G expression in classical HLA class I-negative tumors is of prognostic value for clinical outcome of early breast cancer patients. J Immunol 2010 Dec 15;185(12):7452-9.

(35) Majeti R, Chao MP, Alizadeh AA, Pang WW, Jaiswal S, Gibbs KD, et al. CD47 is an adverse prognostic factor and therapeutic antibody target on human acute myeloid leukemia stem cells. Cell 2009 Jul 23;138(2):286-99.

(36) Serrano M, Lin AW, McCurrach ME, Beach D, Lowe SW. Oncogenic ras provokes premature cell senescence associated with accumulation of p53 and p16INK4a. Cell 1997 Mar 7;88(5):593-602.

(37) Coppe JP, Desprez PY, Krtolica A, Campisi J. The senescence-associated secretory phenotype: the dark side of tumor suppression. Annu Rev Pathol 2010;5:99-118. doi: 10.1146/annurev-pathol-121808-102144.:99-118.

(38) Damgaard CK, Lykke-Andersen J. Regulation of ARE-mRNA Stability by Cellular Signaling: Implications for Human Cancer. Cancer Treat Res 2013;158:153-80.

(39) Moore AE, Chenette DM, Larkin LC, Schneider RJ. Physiological networks and disease functions of RNA-binding protein AUF1. Wiley Interdiscip Rev RNA 2014 Jul;5(4):549-64.

(40) White EJ, Brewer G, Wilson GM. Post-transcriptional control of gene expression by AUF1: mechanisms, physiological targets, and regulation. Biochim Biophys Acta 2013 Jun;1829(67):680-8.

(41) Zinder JC, Lima CD. Targeting RNA for processing or destruction by the eukaryotic RNA exosome and its cofactors. Genes Dev 2017 Jan 15;31(2):88-100.

(42) Laroia G, Cuesta R, Brewer G, Schneider RJ. Control of mRNA decay by heat shock-ubiquitinproteasome pathway. Science 1999 Apr 16;284(5413):499-502.

(43) Hitti E, lakovleva T, Brook M, Deppenmeier S, Gruber AD, Radzioch D, et al. Mitogen-activated protein kinase-activated protein kinase 2 regulates tumor necrosis factor mRNA stability and translation mainly by altering tristetraprolin expression, stability, and binding to adenine/uridinerich element. Mol Cell Biol 2006 Mar;26(6):2399-407.

(44) Clement SL, Scheckel C, Stoecklin G, Lykke-Andersen J. Phosphorylation of tristetraprolin by MK2 impairs AU-rich element mRNA decay by preventing deadenylase recruitment. Mol Cell Biol 2011 Jan;31(2):256-66.

(45) Tiedje C, Diaz-Munoz MD, Trulley P, Ahlfors H, Laaß K, Blackshear PJ, et al. The RNA-binding protein TTP is a global post-transcriptional regulator of feedback control in inflammation. Nucleic Acids Research 2016 Sep 6;44(15):7418-40.

(46) Brooks SA, Blackshear PJ. Tristetraprolin (TTP): interactions with mRNA and proteins, and current thoughts on mechanisms of action. Biochim Biophys Acta 2013 Jun;1829(6-7):666-79.

(47) Shiloh Y, Ziv Y. The ATM protein kinase: regulating the cellular response to genotoxic stress, and more. Nat Rev Mol Cell Biol 2013 Apr;14(4):197-210. 
(48) Tee AR, Proud CG. DNA-damaging agents cause inactivation of translational regulators linked to mTOR signalling. Oncogene 2000 Jun 15;19(26):3021-31.

(49) Jackson SP, Bartek J. The DNA-damage response in human biology and disease. Nature 2009 Oct 22;461(7267):1071-8.

(50) Holohan C, Van Schaeybroeck S, Longley DB, Johnston PG. Cancer drug resistance: an evolving paradigm. Nat Rev Cancer 2013 Oct;13(10):714-26.

(51) Cannell IG, Merrick KA, Morandell S, Zhu CQ, Braun CJ, Grant RA, et al. A Pleiotropic RNABinding Protein Controls Distinct Cell Cycle Checkpoints to Drive Resistance of p53-defective Tumors to Chemotherapy. Cancer Cell 2015 Nov 9;28(5):623-37.

(52) Lalaoui N, Hanggi K, Brumatti G, Chau D, Nguyen NYN, Vasilikos L, et al. Targeting p38 or MK2 Enhances the Anti-Leukemic Activity of Smac-Mimetics. Cancer Cell 2016 Jan 6;29(2):145-58.

(53) Patnaik A, Haluska P, Tolcher AW, Erlichman C, Papadopoulos KP, Lensing JL, et al. A First-inHuman Phase I Study of the Oral p38 MAPK Inhibitor, Ralimetinib (LY2228820 Dimesylate), in Patients with Advanced Cancer. Clin Cancer Res 2016 Feb 29;22(5):1095.

(54) Kuma Y, Sabio G, Bain J, Shpiro N, Marquez R, Cuenda A. BIRB796 inhibits all p38 MAPK isoforms in vitro and in vivo. J Biol Chem 2005 May;\%20;280(20):19472-9.

(55) Grattendick KJ, Nakashima JM, Feng L, Giri SN, Margolin SB. Effects of three anti-TNF-alpha drugs: etanercept, infliximab and pirfenidone on release of TNF-alpha in medium and TNFalpha associated with the cell in vitro. Int Immunopharmacol 2008 May;8(5):679-87.

(56) Nakazato H, Oku H, Yamane S, Tsuruta Y, Suzuki R. A novel anti-fibrotic agent pirfenidone suppresses tumor necrosis factor-a at the translational level. European Journal of Pharmacology 2002 Jun 20;446(1GÇô3):177-85.

(57) Kagoya Y, Yoshimi A, Kataoka K, Nakagawa M, Kumano K, Arai S, et al. Positive feedback between NF-kappaB and TNF-alpha promotes leukemia-initiating cell capacity. J Clin Invest 2014 Feb;124(2):528-42.

(58) Frelin C, Imbert V, Griessinger E, Peyron AC, Rochet N, Philip P, et al. Targeting NF-kB activation via pharmacologic inhibition of IKK2-induced apoptosis of human acute myeloid leukemia cells. Blood 2005 Jan 4;105(2):804.

(59) Chang TP, Vancurova I. Bcl3 regulates pro-survival and pro-inflammatory gene expression in cutaneous T-cell lymphoma. Biochim Biophys Acta 2014 Nov 30;1843(11):2620-30.

(60) Haq R, Yokoyama S, Hawryluk EB, Jönsson GB, Frederick DT, McHenry K, et al. BCL2A1 is a lineage-specific antiapoptotic melanoma oncogene that confers resistance to BRAF inhibition. PNAS 2013 Mar 12;110(11):4321-6.

(61) Kurosu T, Fukuda T, Miki T, Miura O. BCL6 overexpression prevents increase in reactive oxygen species and inhibits apoptosis induced by chemotherapeutic reagents in B-cell lymphoma cells. Oncogene 2003 Jan 5;22(29):4459-68. 
(62) Rushworth SA, Bowles KM, Raninga P, MacEwan DJ. NF-kappaB-inhibited acute myeloid leukemia cells are rescued from apoptosis by heme oxygenase-1 induction. Cancer Res 2010 Apr 1;70(7):2973-83.

(63) Emmons J, Townley-Tilson WHD, Deleault KM, Skinner SJ, Gross RH, Whitfield ML, et al. Identification of TTP mRNA targets in human dendritic cells reveals TTP as a critical regulator of dendritic cell maturation. RNA 2008 May;14(5):888-902.

(64) Kondoh K, Nishida E. Regulation of MAP kinases by MAP kinase phosphatases. Biochimica et Biophysica Acta (BBA) - Molecular Cell Research 2007;1773(8):1227-37.

(65) Li ZW, Dalton WS. Tumor microenvironment and drug resistance in hematologic malignancies. Blood Reviews 2006;20(6):333-42.

(66) Townsend E, Murakami M, Christodoulou A, Christie A, K+|ster J, Desouza T, et al. The Public Repository of Xenografts Enables Discovery and Randomized Phase II-like Trials in Mice. Cancer Cell30(1):183.

(67) Sykes DB, Kfoury YS, Mercier FE, Wawer MJ, Law JM, Haynes MK, et al. Inhibition of Dihydroorotate Dehydrogenase Overcomes Differentiation Blockade in Acute Myeloid Leukemia. Cell 2016 Sep 22;167(1):171-86.

(68) Sosa MS, Avivar-Valderas A, Bragado P, Wen HC, Aguirre-Ghiso JA. ERK1/2 and p38a/b Signaling in Tumor Cell Quiescence: Opportunities to Control Dormant Residual Disease. Clin Cancer Res 2011 Sep 15;17(18):5850-7.

(69) Claudio N, Dalet A, Gatti E, Pierre P. Mapping the crossroads of immune activation and cellular stress response pathways. EMBO J 2013 May 2;32(9):1214-24.

(70) Senft D, Ronai ZA. UPR, autophagy, and mitochondria crosstalk underlies the ER stress response. Trends Biochem Sci 2015 Mar;40(3):141-8.

(71) Cuenda A, Rousseau S. p38 MAP-Kinases pathway regulation, function and role in human diseases. Biochimica et Biophysica Acta (BBA) - Molecular Cell Research 2007 Aug;1773(8):1358-75.

(72) Korb A, Tohidast-Akrad M, Cetin E, Axmann R, Smolen J, Schett G. Differential tissue expression and activation of p38 MAPK alpha, beta, gamma, and delta isoforms in rheumatoid arthritis. Arthritis \& Rheumatism 2006 Sep 1;54(9):2745-56.

(73) Liu J, Lin A. Role of JNK activation in apoptosis: A double-edged sword. Cell Research 2005 Jan 1;15:36.

(74) Nichols RC, Botson J, Wang XW, Hamilton BJ, Collins JE, Uribe V, et al. A flexible approach to studying post-transcriptional gene regulation in stably transfected mammalian cells. Mol Biotechnol $2011 \mathrm{Jul} ; 48(3): 210-7$.

(75) Zhang T, Kruys V, Huez G, Gueydan C. AU-rich element-mediated translational control: complexity and multiple activities of trans-activating factors. Biochem Soc Trans 2001 $\mathrm{Dec} ; 30(6): 952-8$. 
(76) Mukherjee N, Corcoran DL, Nusbaum JD, Reid DW, Georgiev S, Hafner M, et al. Integrative Regulatory Mapping Indicates that the RNA-Binding Protein HuR Couples Pre-mRNA Processing and mRNA Stability. Mol Cell 2011 Aug 5;43(3):327-39.

(77) Lal A, Mazan-Mamczarz K, Kawai T, Yang X, Martindale JL, Gorospe M. Concurrent versus individual binding of HuR and AUF1 to common labile target mRNAs. EMBO J 2004 Aug 4;23(15):3092-102.

(78) Kesarwani M, Kincaid Z, Gomaa A, Huber E, Rohrabaugh S, Siddiqui Z, et al. Targeting c-FOS and DUSP1 abrogates intrinsic resistance to tyrosine-kinase inhibitor therapy in BCR-ABLinduced leukemia. Nature Medicine 2017 Mar 20;23:472.

(79) Coelho MA, de Carn+ $+\operatorname{Tr}+\neg$ cesson S, Rana S, Zecchin D, Moore C, Molina-Arcas M, et al. Oncogenic RAS Signaling Promotes Tumor Immunoresistance by Stabilizing PD-L1 mRNA. Immunity 2017 Dec 19;47(6):1083-99. 
Figure 1. G0 leukemic cells induced by AraC or serum-starvation are chemoresistant and recapitulate gene expression programs of in vivo chemoresistant and G0 models. A.

Transcriptome, translatome and proteome analyses in proliferating and G0 leukemic cells. G0 cells (AraCS, SS cells) were induced by treatment of proliferating cells (S+) with AraC or serum starvation. Total RNAs, polysome-associated mRNAs and protein were analyzed by comparative microarray and quantitative proteomics. B. Polysome profiles of S+, SS and AraCS are shown. Polysome-associated mRNAs were isolated and analyzed by microarray. C. Ki67 translatome level and flow cytometric quantification of G0/G1, S and G2/M phases, using BrdU and PI staining. D. Cell counting with trypan blue staining. Proliferating THP1 cells were serum-starved or treated with AraC for days specified. Then, serum was added to SS cells while AraCS cells were resuspended in fresh media. E. S+, SS and AraCS cells were treated with various concentration of AraC for 3 days. Viable THP1 leukemic cells were measured by cell counting using trypan blue staining and $\mathrm{IC}_{50}$ values of AraC are shown. $\mathbf{F}$. Comparison of transcriptomic, translatomic and proteomic changes in response to SS and $5 \mu \mathrm{M}$ AraC treatments. G. Comparison of AraCS and SS with leukemic stem cells (LSC) (16) in AML, dormant leukemic cells (LRC) (15), minimal residual disease (MRD) (15) in ALL, and G0 fibroblasts (1). GSEA analysis was performed to determine whether previously published transcriptome signatures of LSC, LRC, MRD and GO HFF are up-regulated in AraCS and SS cells, compared to S+ cells. 'N' marks the limited resolution of the proteome in the GSEA. * $P \square \leq \square 0.05$. Data are represented as average \pm SEM. See also Fig. S1 \& Table S1.

Figure 2. Inflammatory response mRNAs are selectively translated in Go leukemic cells, where canonical translation is inhibited. A. Repression of canonical translation. B. Polysome to monosome ratios in $\mathrm{S}_{+}, \mathrm{SS}$ and AraCS. C. Western analysis of translation initiation factor, elF2 $\alpha$ and its regulators elF4EBP, PERK and PKR. D. Boxplot of the transcriptome and translatome changes in known TOP mRNAs in response to SS or AraC treatment. E. Number of differentially expressed genes. F. Venn 
diagram shows 162 genes up-regulated at both the transcriptome and translatome levels and 180 genes translationally up-regulated, where ribosome occupancy, RO increases by at least 1.5-fold. Heatmap shows gene expression changes in RNA, translatome levels and RO. G. Gene ontology (GO) analyses of differentially expressed genes shown in Fig. 2E. Statistical significance of enriched GO categories is shown as a heatmap. $\mathbf{H}$. Expression of signature genes of G0 leukemic cells in published transcriptomes of in vivo resistant leukemic and G0 models in other Gocells. I. Translatome analysis of G0 cells from five different cell types. Heatmap of normalized enrichment score (NES) is shown. ${ }^{*} P \square \leq \square 0.05$. Data are represented as average \pm SEM. See also Fig. S2 \& Table S1.

Figure 3. Phosphorylation of TTP stabilizes ARE-bearing TNFa in G0 leukemic cells. A. Boxplot of ARE scores (SI methods) in the 3'UTRs of genes which are up- or down-regulated at the translatome or RNA levels in G0 compared to S+ cells. B. Venn diagram shows genes that are up-regulated at the translatome level and contain AREs (left). List of such genes (right, Table S2). C. Expression of ARE genes at the RNA and translatome levels. D. Scatter plot showing the expression of RNA binding protein genes from RBPDB database (SI methods). TTP is indicated with a green dot. E. Western analysis of TTP in lysates from multiple leukemic cell lines in the absence or presence of alkaline phosphatase (AP). Phospho-TTP is indicated with an arrow. F. Bar graph shows TNFa mRNA expression normalized to GAPDH mRNA upon over-expression of vector or c-myc tagged nonphosphorylatable mutant TTP (TTP-AA) in AraC-treated THP1 or K562 cells. Western analysis of TTPAA with c-myc antibody (right). G. Half-life of TNFa mRNA. TTP-deficient BMDM cells were transduced with doxycycline inducible plasmids that express GFP vector, TTP wild-type or TTP-AA mutant. Cells were induced with $1 \mu \mathrm{g} / \mathrm{ml}$ doxycycline prior to $1 \mu \mathrm{M} \mathrm{AraC}$ treatment. Western analysis of induction of TTP protein. TNFa mRNA level was measured at indicated time points by qPCR after transcriptional arrest with $5 \mu \mathrm{g} / \mathrm{ml}$ actinomycin D treatment. H. Association of TTP-AA with TNFa mRNA in AraCS cells. TTP-AA was immunoprecipitated with GFP antibody from AraC-treated BMDM cells expressing GFP- 
tagged TTP-AA. (Western blot), followed by qPCR analysis of TNFa mRNA (graph). ${ }^{*} P \square \leq \square 0.05$. Data are represented as average \pm SEM. See also Fig. S3 \& Table S2.

\section{Figure 4. Phosphorylation of TTP by p38 MAPK-MK2 promotes chemoresistance}

A. The p38 MAPK (p38)-MK2 pathway enables stabilization and translation of ARE-bearing mRNAs via TTP phosphorylation in chemoresistant G0 cells. LY2228820 (LY) and BIRB396 (BB) are p38 inhibitors.

B. Western analysis of in lysates from THP1 cells at indicated time points after AraC treatment. C.

Western analysis in S+ and AraCS cells treated with vehicle, $5 \mu \mathrm{M} L Y$ or $5 \mu \mathrm{M} B B$. D. Firefly luciferase activity of a reporter bearing TNFa ARE in its 3'UTR normalized to activity of co-transfected Renilla luciferase in S+ and AraCS cells treated with vehicle, $5 \mu \mathrm{M}$ LY. E. Sequential treatment with p38 inhibitors and AraC in leukemic cells. F-G. Effect of p38 inhibitions on survival of AraC-resistant cells. THP1 cells were treated with $5 \mu \mathrm{M} \mathrm{BB}, 5 \mu \mathrm{M} L Y$, and vehicle in the absence (S+, top panels) or presence (AraC, bottom panels) of $5 \mu \mathrm{M}$ AraC treatment for three days. Bar graphs show relative cell viability and death assessed by cell counting, MTS and caspase 3/7 assays. In the presence of AraC, THP1 cells were treated with p38 inhibitors prior to AraC treatment $(\mathrm{BB} \rightarrow \mathrm{AraC}, \mathrm{LY} \rightarrow \mathrm{AraC})$, at the same time with $\operatorname{AraC}(\mathrm{AraC}+\mathrm{BB})$ and 1 day after $\operatorname{AraC}(\mathrm{AraC} \rightarrow \mathrm{BB}, \mathrm{AraC} \rightarrow \mathrm{LY}) .4 \mathrm{H}$ and $1 \mathrm{D}$ indicate 4 hours and 1 days, respectively. H-I. Effect of TTP-AA mutant on survival of AraC resistant cells. TTPAA mutant expression prior to $5 \mu \mathrm{M}$ AraC treatment, decreased TNFa in THP1 or K562 cells (Fig. 3F). Cell viability was assessed by cell count $(\mathrm{H})$. TTP-AA, TTP wild-type and vector were expressed in TTP-deficient BMDM cells prior to $1 \mu \mathrm{M}$ AraC treatment. Bar graphs show relative cell viability and death (I). J. Effect of p38 inhibition on resistant cells from five AML cell lines (M5 FAB subtype). Cells were treated with $5 \mu \mathrm{M} L Y$ or vehicle 4 hours prior to AraC treatment (top panel, AraC) or in the absence of AraC (bottom panel, S+). Human CD34+ cells from healthy donors were tested as a control. K. Effect of p38 inhibition on survival of chemoresistant cells induced with various concentrations of 
AraC. MV4:11 leukemic cells were treated with $5 \mu \mathrm{M} L Y$ or vehicle prior to $0 \mu \mathrm{M}, 0.2 \mu \mathrm{M}, 0.5 \mu \mathrm{M}$ or 1 $\mu \mathrm{M}$ AraC for 3 days. ${ }^{*} P \square \leq \square 0.05$. Data are represented as average \pm SEM. See also Fig. S4.

\section{Figure 5. TNFa induced by phosphorylation of TTP promotes chemoresistance}

A. Phosphorylation of TTP by the p38-MK2 pathway stabilizes ARE-bearing TNFa mRNA, resulting in activation of NF-kB signaling in resistant G0 leukemic cells. TNFa expression is inhibited by TTP-AA mutant, pirfenidone (PFD) or shRNAs, and NF-kB signaling by NF-kB inhibitor, Bay11-7082. B.

Expression of TNFa and NF-kB target genes at the translatome level at indicated time points after SS or AraC treatment. C. TNFa protein level in S+, SS and AraCS cells. D. Effect of TNFa on chemoresistance. THP1 cells were transduced with doxycycline inducible shRNA against TNFa or control shRNA. ShRNA against TNFa was induced prior to AraC (shTNFa $\rightarrow$ AraC) or after AraC (AraC $\rightarrow$ shTNFa) and recombinant TNFa protein was added 1 day prior to AraC (ReTNFa $\rightarrow$ AraC). Cell viability and western analysis of TNFa, are shown. E. Effect of PFD on TNFa expression at the translatome (middle) and protein levels (right) in AraCS cells. F. Effect of pharmacological inhibition of TNFa by PFD on AraC resistance. THP1 cells were treated with $300 \mu \mathrm{g} / \mathrm{ml}$ PFD or vehicle in the absence of $\mathrm{AraC}(\mathrm{S}+$, top panels), in the presence of $\operatorname{AraC}$ (AraC, middle panels), or on serum starvation (SS, bottom panels). Bar graphs show cell viability and death assessed by cell counting, MTS and caspase 3/7 assays. In middle or bottom panels, THP1 cells were treated with PFD 1 day prior to AraC or SS (PFD $\rightarrow$ AraC, PFD $\rightarrow$ SS), at the same time with AraC or SS (AraC + PFD, SS + PFD), and 1 day after AraC or SS (AraC $\rightarrow$ PFD, SS $\rightarrow$ PFD). G. Effect of TNFa inhibition on AraC resistance from six different leukemic cell lines. Cells were treated with PFD or vehicle 1 day prior to AraC (AraC, top panels) or in the absence of AraC (bottom panels, S+). H. Effect of NF-kB inhibition on AraC resistance. THP1 cells were treated with $10 \mu \mathrm{M}$ Bay11-7082 or vehicle in the absence of AraC (S+, top panels), in the presence of AraC (AraC, middle panels) or under serum starvation (SS, bottom panels). In middle or bottom panels, THP1 cells were treated with Bay11-7082 1 day prior to AraC or 
SS (Bay $\rightarrow$ AraC, Bay $\rightarrow$ SS), at the same time with AraC or SS (AraC + Bay, SS + Bay), and 1 day after AraC or SS (AraC $\rightarrow$ Bay, SS $\rightarrow$ Bay). ${ }^{\star} P \square \leq \square 0.05$. Data are represented as average \pm SEM. See also Fig. S5.

\section{Figure 6. TTP regulates a pro-apoptotic JNK pathway via targeting DUSP1}

A. Phosphorylation of TTP allows expression of the ARE-bearing mRNA of DUSP1 that inhibits JNK, and hence blocks JNK-mediated apoptosis. JNK pathway is blocked by the inhibitor JNK-IN-8. B-D. Effect of TTP-AA mutant on DUSP1 and phosphorylation of JNK. BMDM TTP deficient cells were treated with doxycycline to express TTP-AA and TTP wild-type prior to AraC treatment. DUSP1 mRNA level was measured by qPCR and is shown relative to GAPDH mRNA (B). Western analysis of TTP, DUSP1 and phospho-JNK is shown (C). TTP-AA was immunoprecipitated with GFP antibody, followed by qPCR analysis for DUSP1 mRNA (D). E. Western analyses in THP1 and MOLM13 cells treated with indicated drug combinations. Phospho-TTP is indicated with an arrow and quantitation of TNFa protein is shown below. F. JNK pathway mediates apoptosis. MOLM13 cells treated with indicated drug combinations. JNK pathway was inhibited with $1 \mu \mathrm{M}$ JNK-IN-8. Western analyses of phosphoJNK, phospho-C-Jun and c-Jun shown on the left; associated cell viability and death graphed on the right. Data are represented as average \pm SEM.

\section{Figure 7. PLA therapy decreases AraC-resistant cells in AML cell lines}

A. PLA therapy, involves pre-treatment of leukemic cells with $\underline{P F D}$ and $\underline{L} Y$ followed by $\underline{\text { AraC }}$ treatment, using half of the concentrations used for individual drugs in Fig. 4 and 5. B. Three different AML cell lines were sequentially treated with indicated drugs, followed by assessment of cell viability and death. C-D. Viability of MOLM13 cells treated with indicated drug combinations. Flow cytometric profiles of cells stained with annexin $\mathrm{V}$ and propidium iodide are shown $(\mathrm{C})$. Cells were plated on methylcellulose media to test colony formation in the presence of drug combinations. Representative 
colony images and quantification of colonies are shown (D). ${ }^{*} \square \leq \square 0.05$. Data are represented as average \pm SEM. See also Fig. S5.

Figure 8. PLA therapy significantly reduces AraC resistance in primary AML cells ex vivo and in vivo. A. Viability of primary cells from AML patients and normal CD34+ cells from healthy donors after indicated treatments. B. Viability and death of primary cells from AML mouse models driven by HoxA9-Meis1 and MLL-AF9 after indicated treatments. C-G. Bioluminescence images and quantification of tumor growth in NSG mice engrafted with MOLM13 cells and treated with PLA therapy or AraC (C-D) and in C57BL/6 mice engrafted with primary HoxA9-Meis1/luciferase cells and treated with PLA therapy or AraC (E-F) or treated with PFD plus LY or vehicle as a control (G). $\mathbf{H}$. Kaplan-Meier survival curves of MLL-AF9 engrafted C57BL/6 mice, treated with PLA therapy or AraC. ${ }^{*} P \square \leq \square 0.05$. Data are represented as average \pm SEM. See also Fig. S5-S6. 
A

Proliferating leukemic cells $(\mathrm{S}+)$

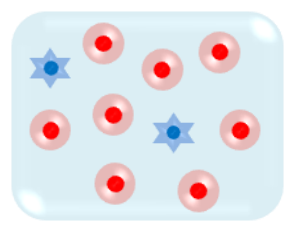

\section{Serum starvation} or Cytarabine

Quiescent, Chemo-resistant leukemic cells (SS, AraCS)

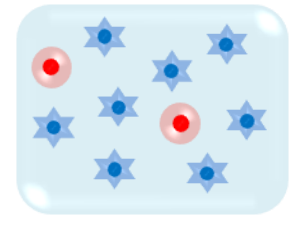

D

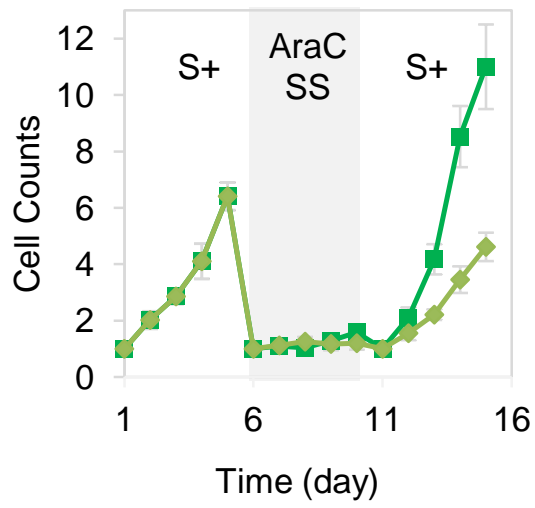

E

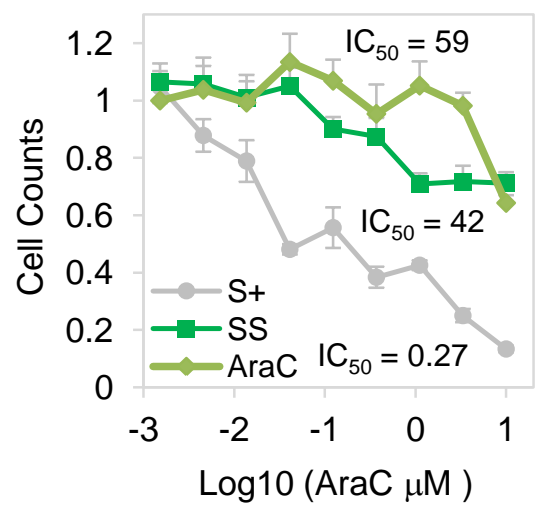

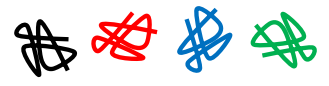

Total proteins

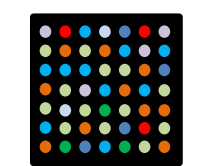

Microarray analysis

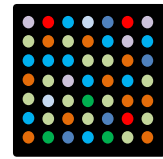

Polysome-bound mRNAs (Translatome)

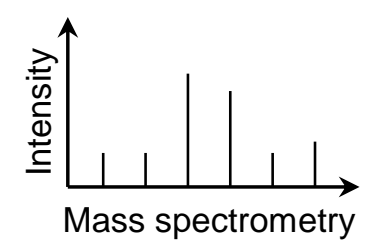

B

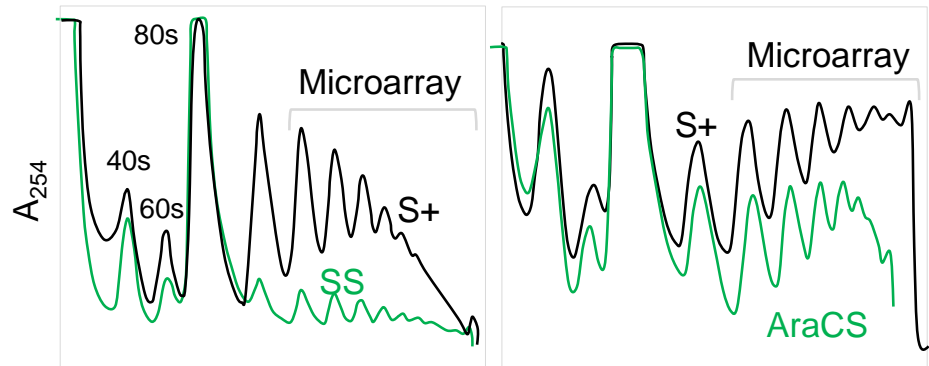

C

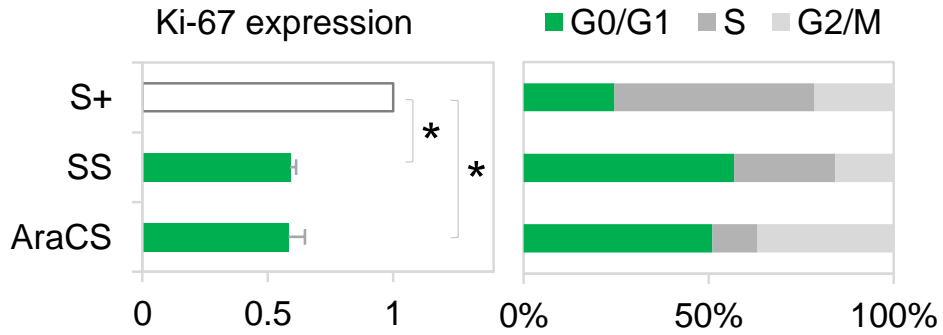

G

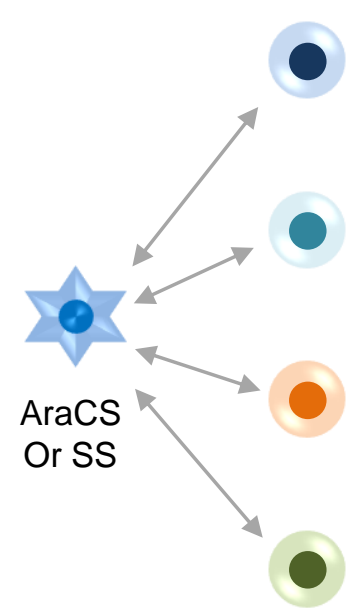

Leukemia stem cells (LSC)

(Saito et al., 2010)

Dormant leukemic cells (LRC)

(Ebinger et al., 2016)

Minimal residual disease (MRD)

(Ebinger et al., 2016)

GO HFF

(Coller et al., 2006)
$\mathrm{F}$

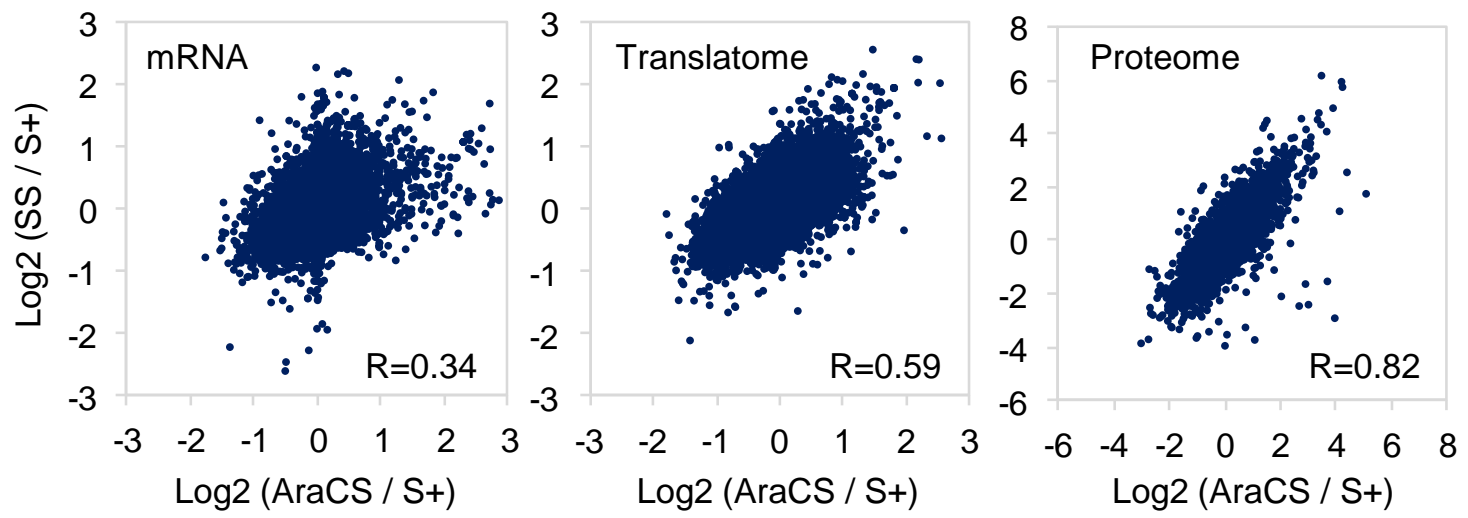

AraCS vs S+ cells
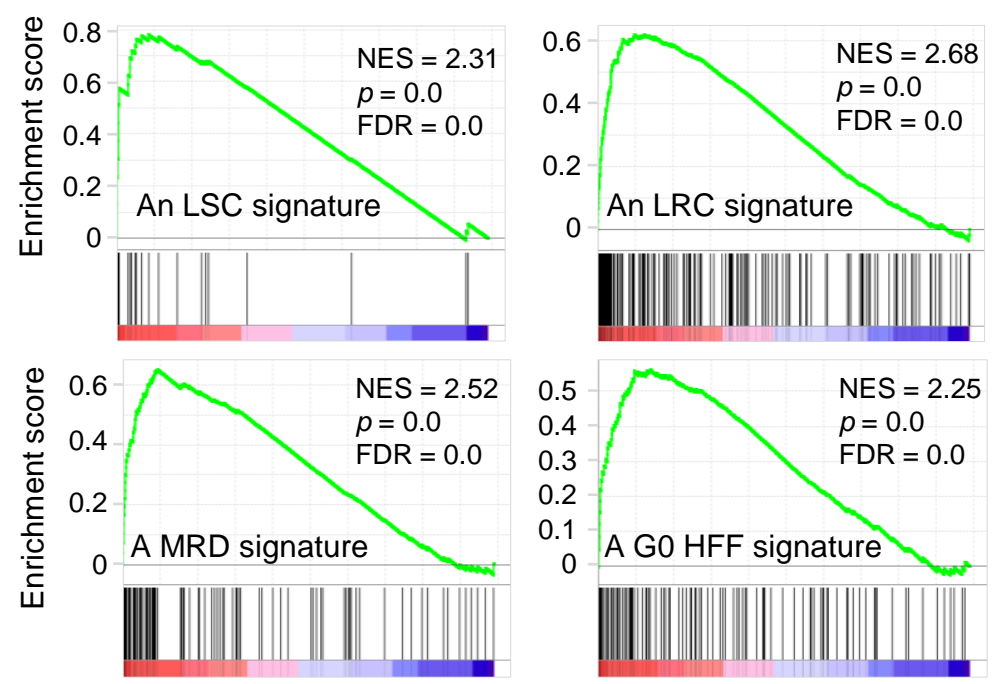

AraCS or SS vs $\mathrm{S}+$ cells

Translatome Proteome

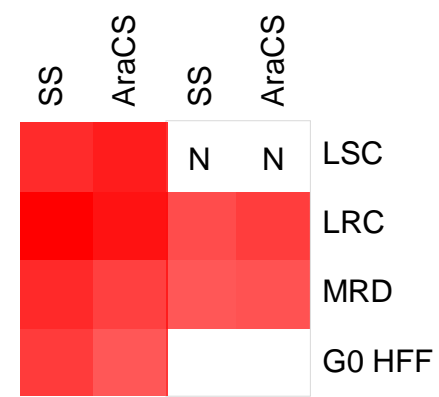

NES

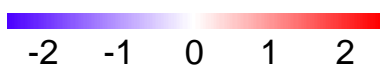



aCC-BY-NC-ND 4.0 International license.

A

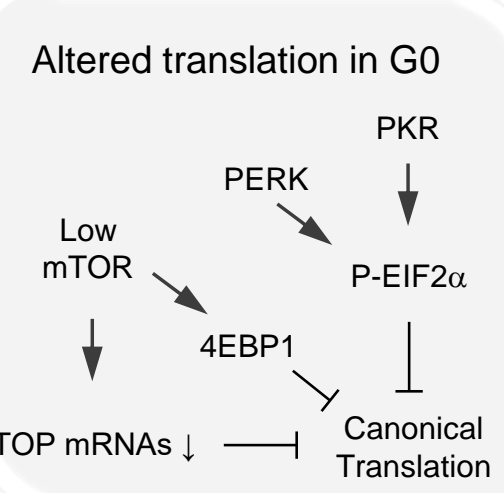

$\mathrm{E}$

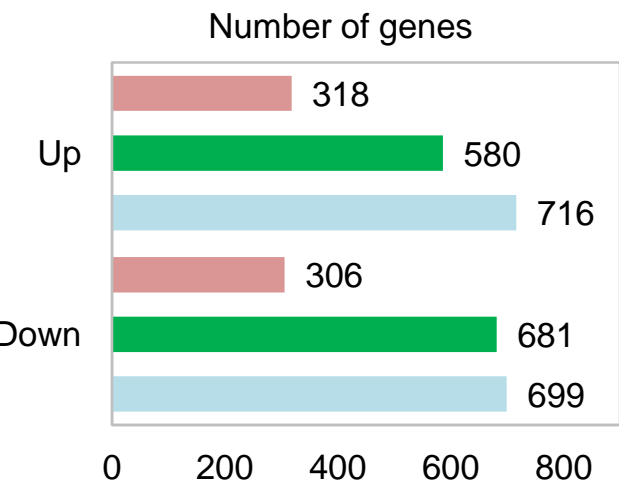

G

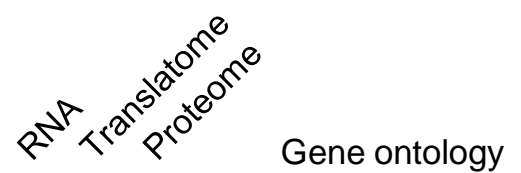

우

కุ Immune response Inflammatory response Cell adhesion Cell migration MAPKKK Translation rRNA processing Ribosome biogenesis
B

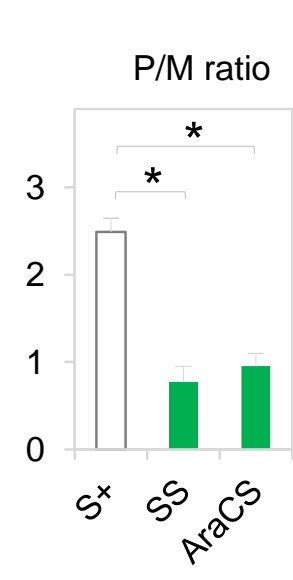

C

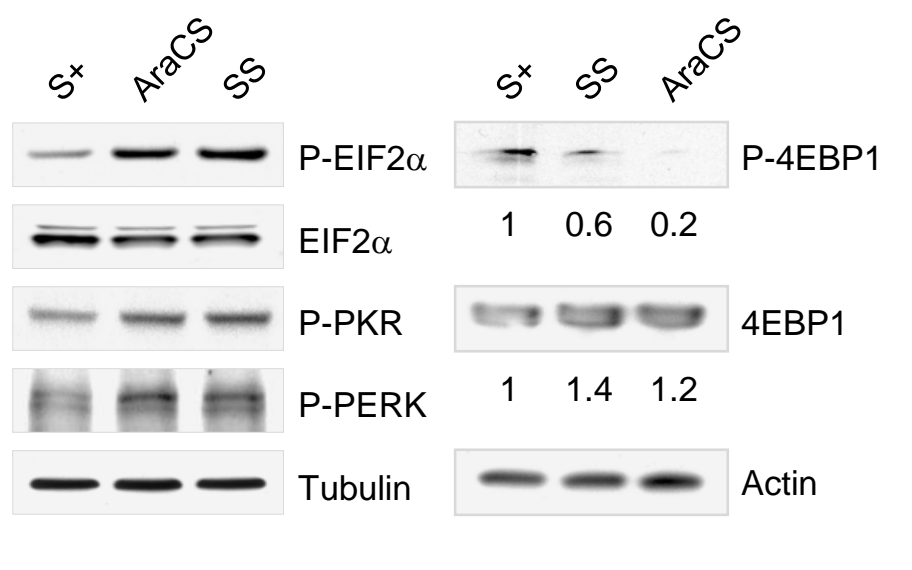

F

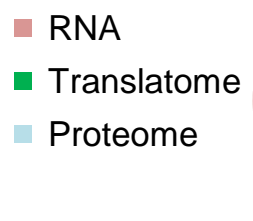

$\mathrm{H}$

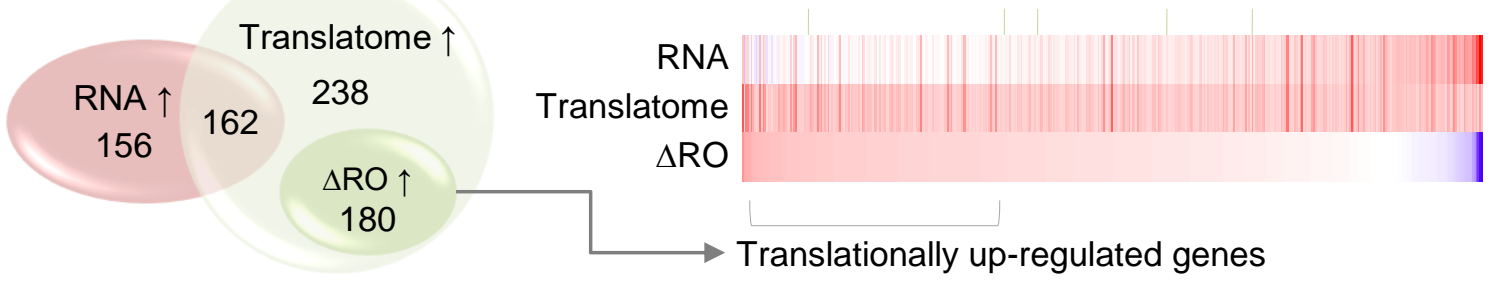
Antigen processing and presentation

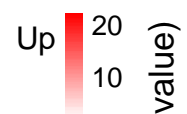

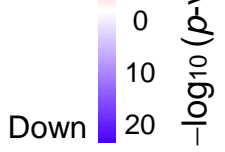
Cell cycle

\section{Expression of signature genes of G0 leukemic cells}

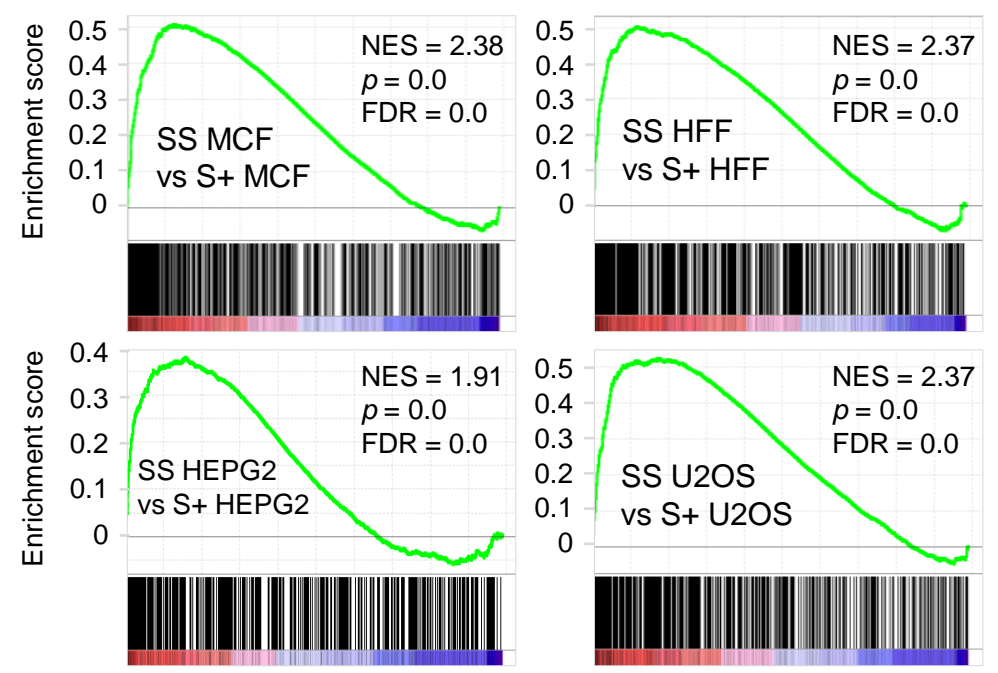

Known TOP mRNAs

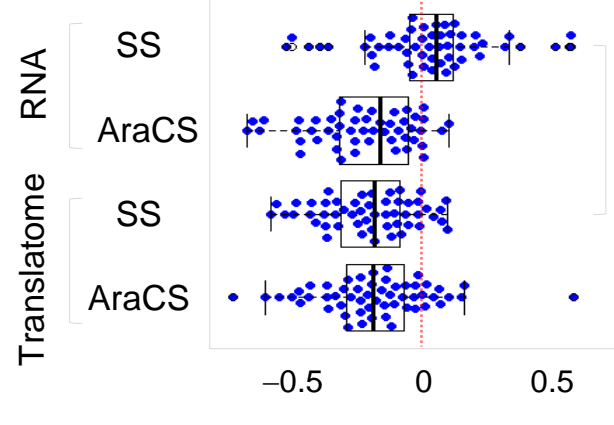

Log2 (AraC or SS / S+)
立㟔皆京

SS vs S+ cells

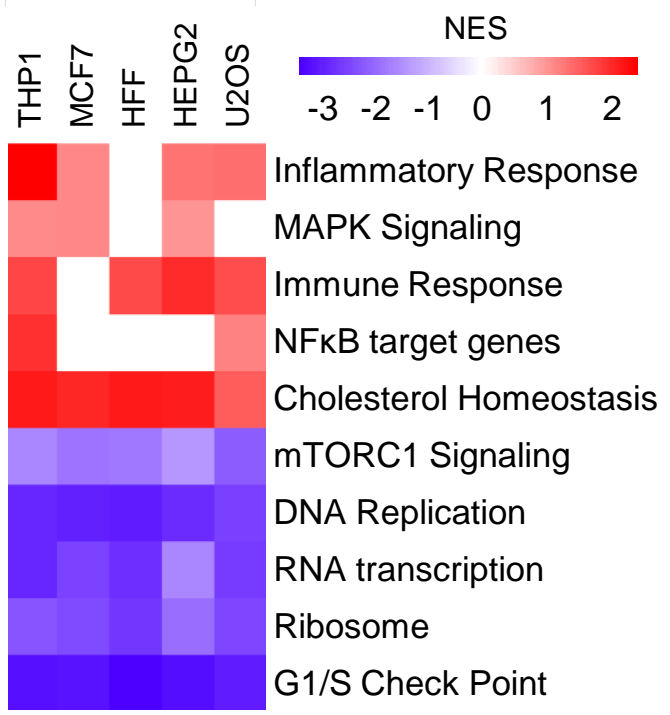


A

C

$E$

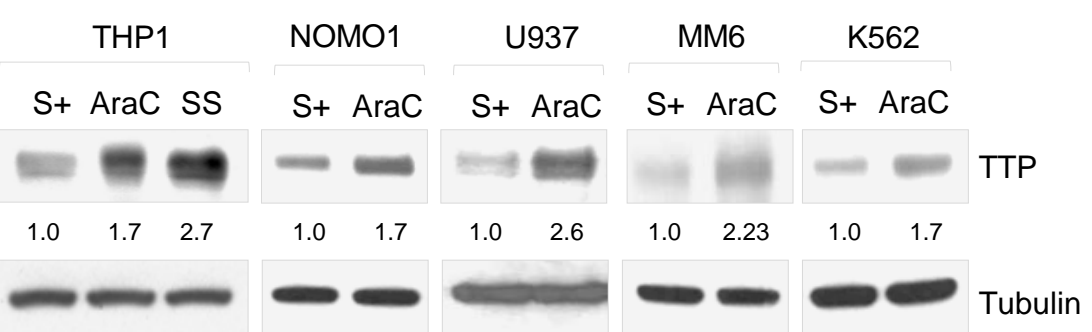

G

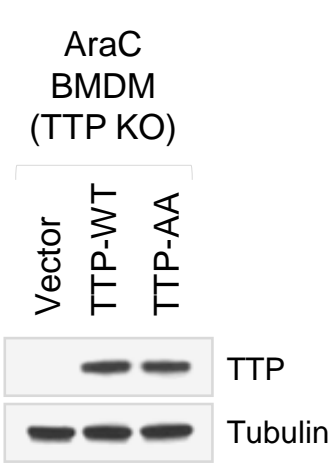

The scores of AU-rich elements

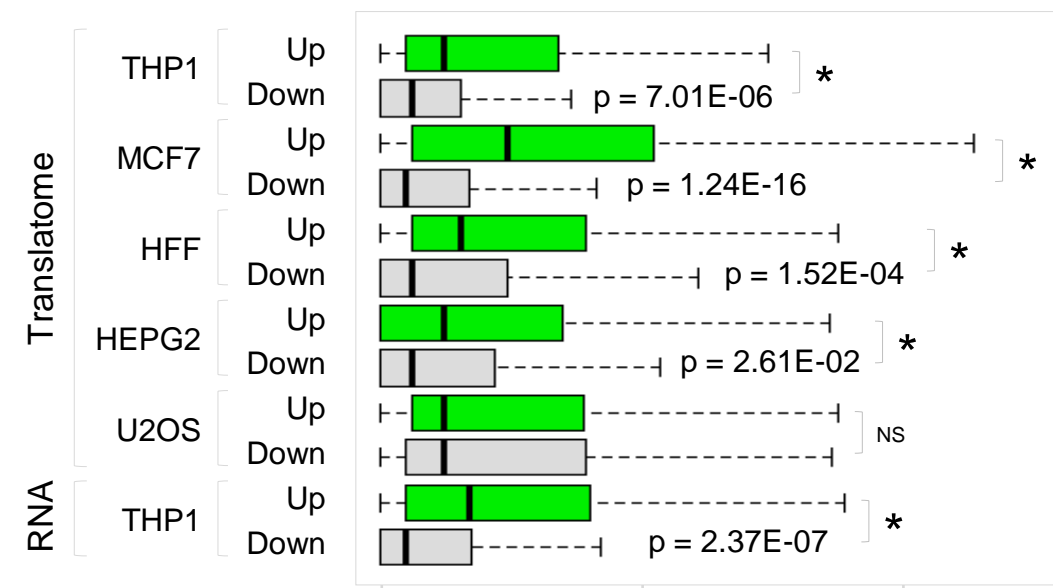

$\begin{array}{lll}0 & 10 & 20\end{array}$

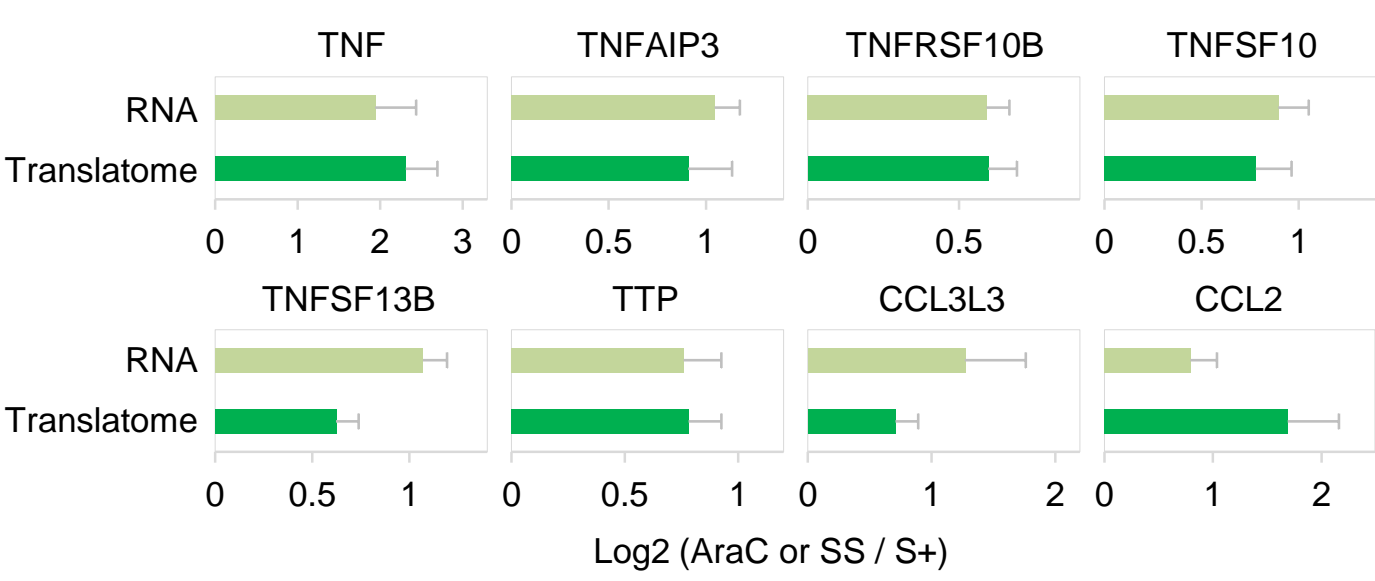

S+ AraC SS SS/AP
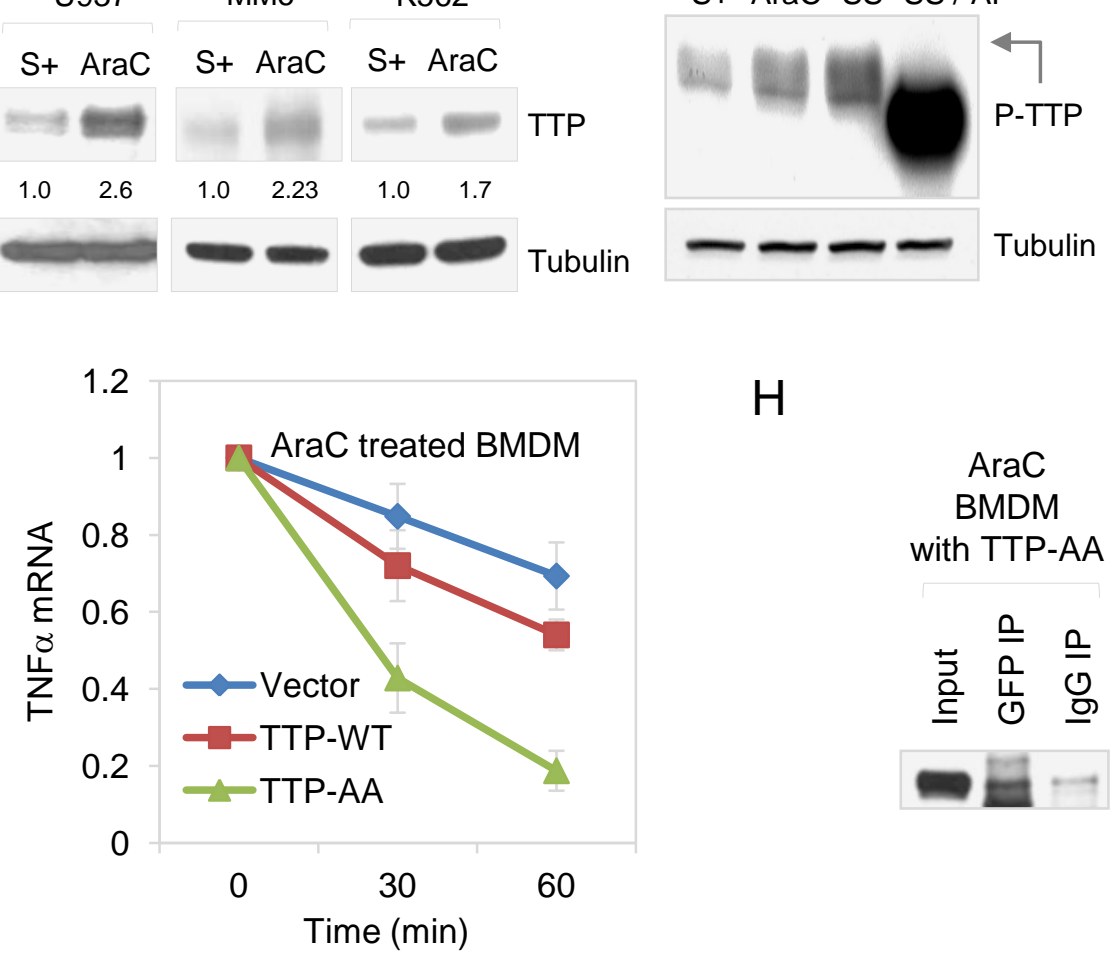

THP1

$\mathrm{H}$

ARE genes up-regulated at the translatome level

\begin{tabular}{clcc}
\hline Gene symbol & AU-rich elements & Start site & Stop site \\
\hline TNF & ...ATTTATTTATTTA... & 482 & 495 \\
TNFAIP3 & ...CTTTATTTATTTT... & 986 & 999 \\
TNFRSF10B & $\ldots$...TTATTTATTTATT... & 345 & 358 \\
TNFSF10 & $\ldots$..TCATATTTATTGT... & 833 & 846 \\
TNFSF13B & ...CTTTATTTAAATA... & 1340 & 1353 \\
TTP & ...CTTTATTTATTCT... & 504 & 517 \\
CCL3L3 & ...TAATTTATTTATA... & 192 & 205 \\
CCL2 & ...TCTTATTTAAGTT... & 168 & 181 \\
\hline
\end{tabular}

D

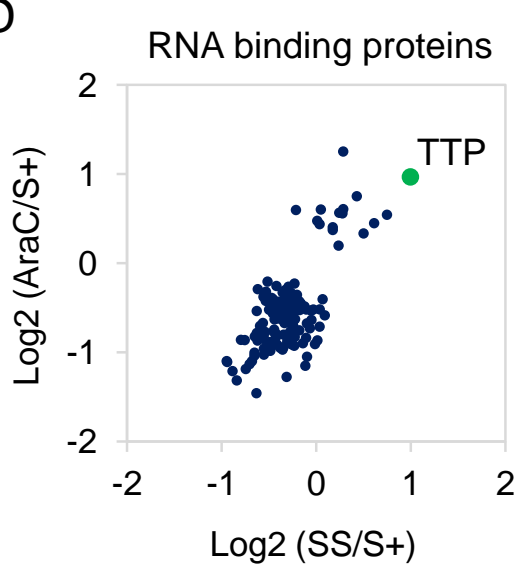

$\mathrm{F}$
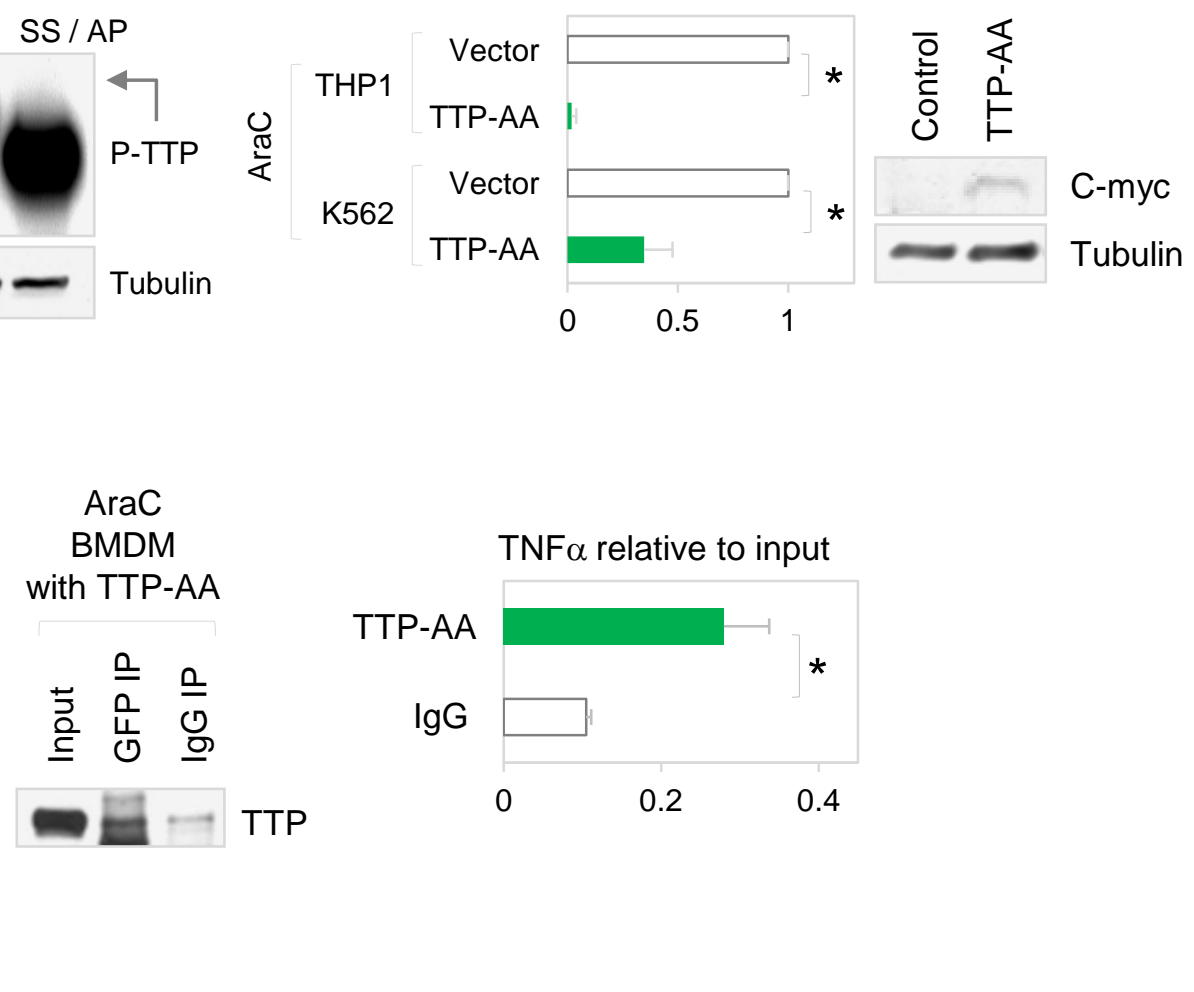
A

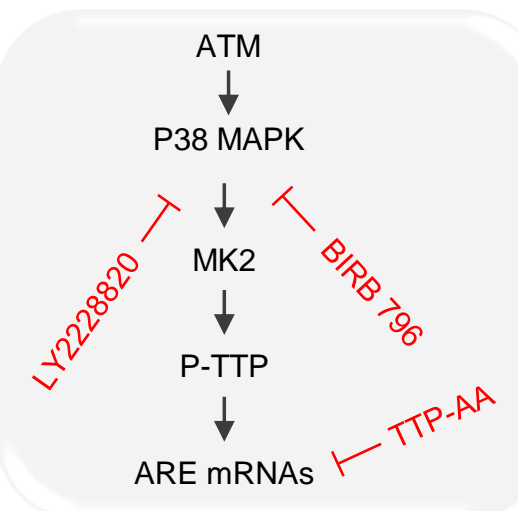

E

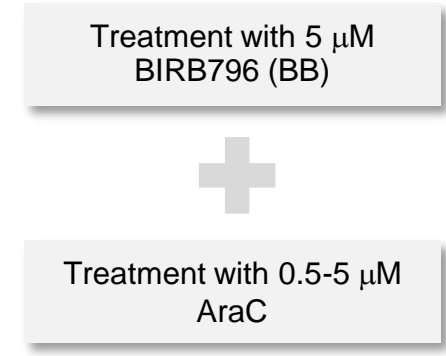

G

ì

$\underset{\frac{\pi}{\alpha}}{\mathbb{\alpha}}$

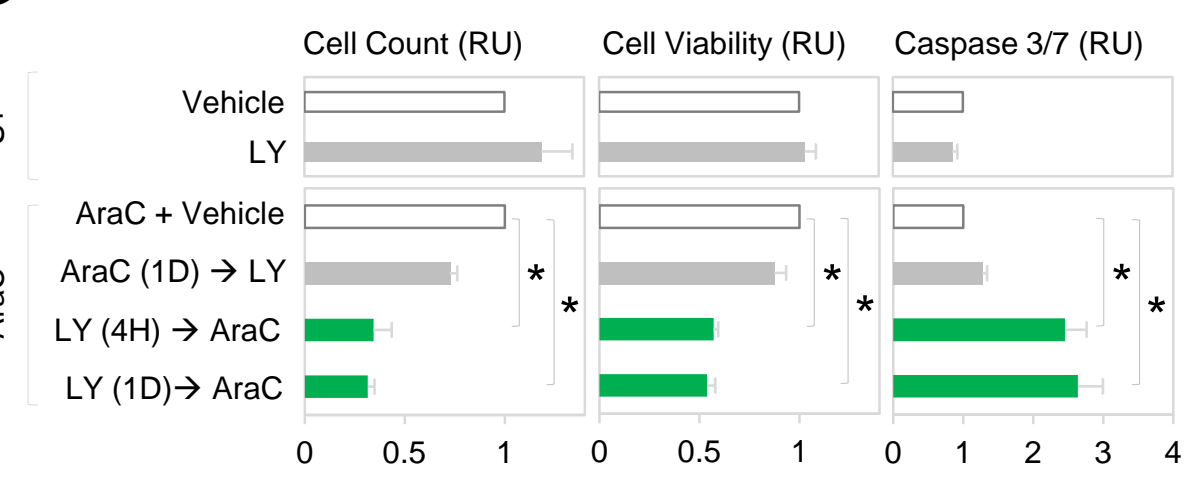

I
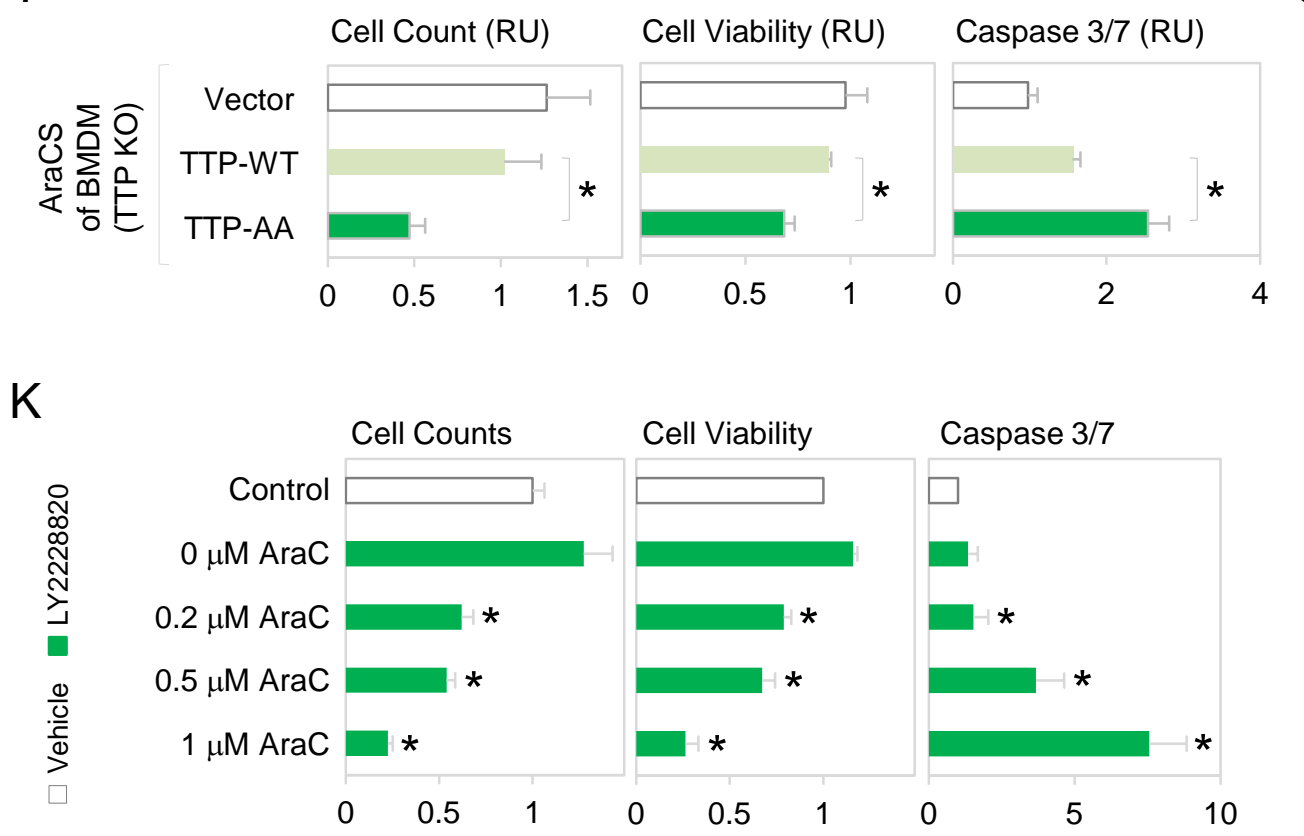

C

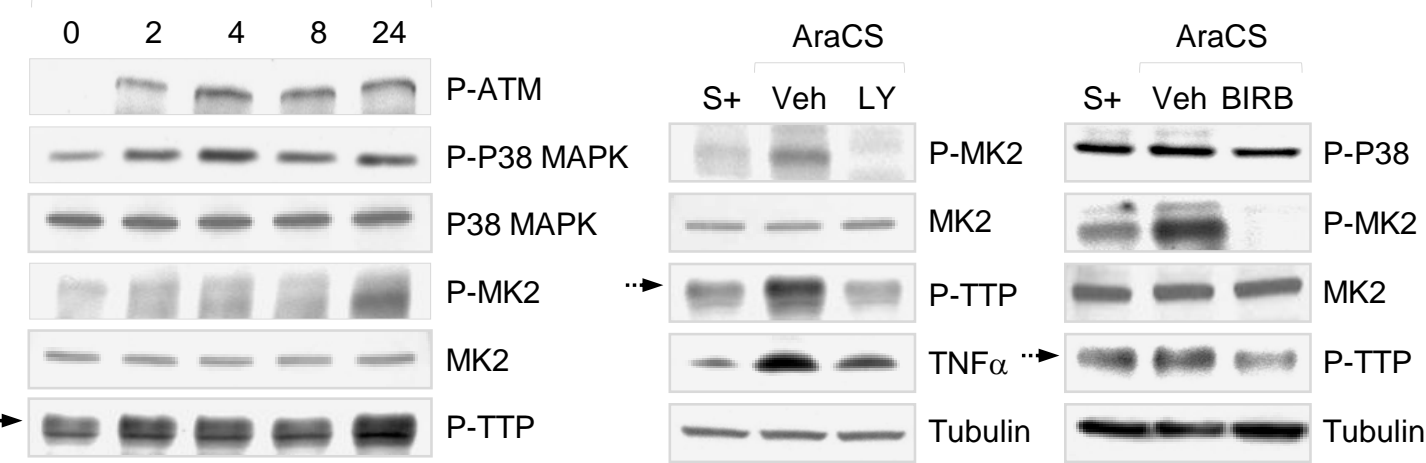

D

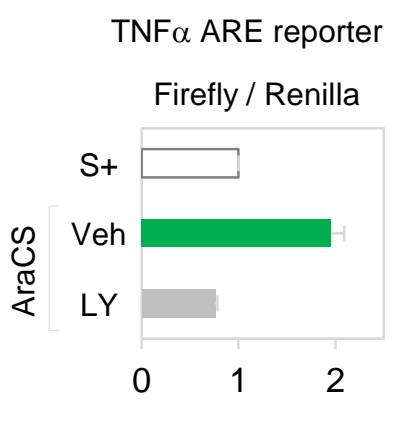

$F$
+
+
0
0
$\frac{\pi}{4}$

$\mathrm{H}$

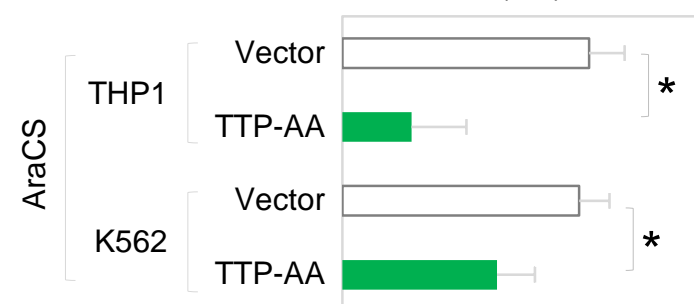

$J$

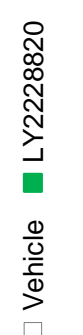
Vehicle
$\mathrm{BB}$
$\mathrm{AraC}+$ Vehicle
$\mathrm{AraC}(1 \mathrm{D}) \rightarrow \mathrm{BB}$
$\mathrm{AraC}+\mathrm{BB}$ $\mathrm{BB}(4 \mathrm{H}) \rightarrow \mathrm{AraC}$

Cell Count (RU) Cell Viability (RU) Caspase $3 / 7$ (RU)

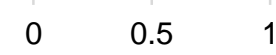

Cell Count $(\mathrm{RU}) \quad$ Cell Viability $(\mathrm{RU}) \quad$ Caspase 3/7 (RU)

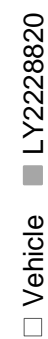

O MM6 $=$ *

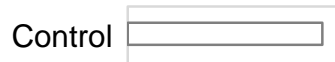

THP1 *

MOLM13

MV4:11

NOMO1

CD34+

Control

THP1

MM6

市

MOLM13

MV4:11

NOMO1

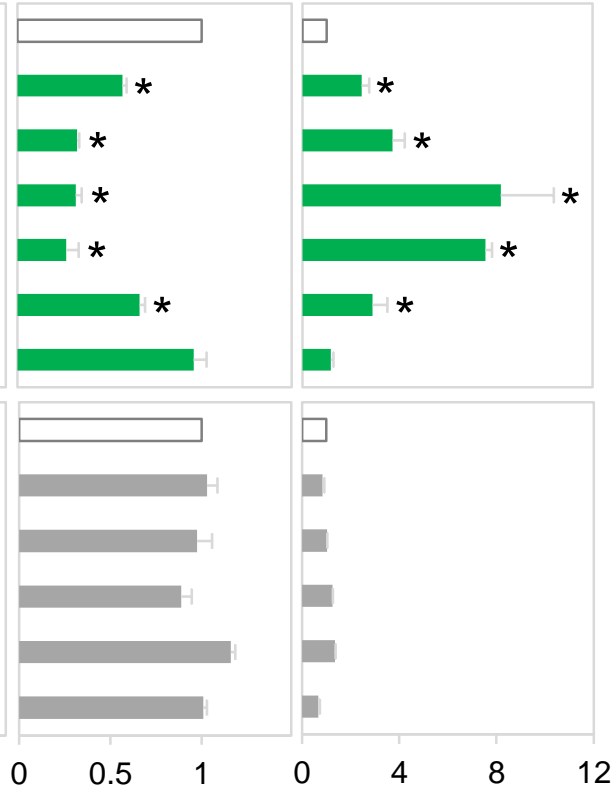




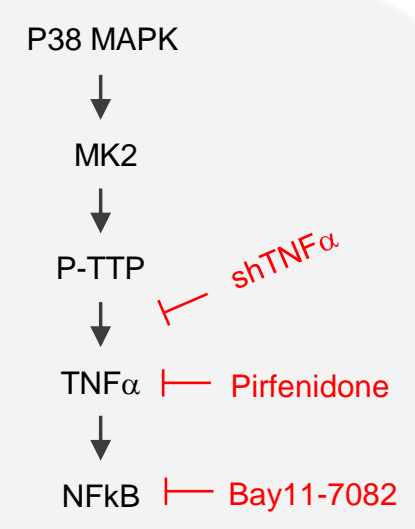

D

D

$E$

F
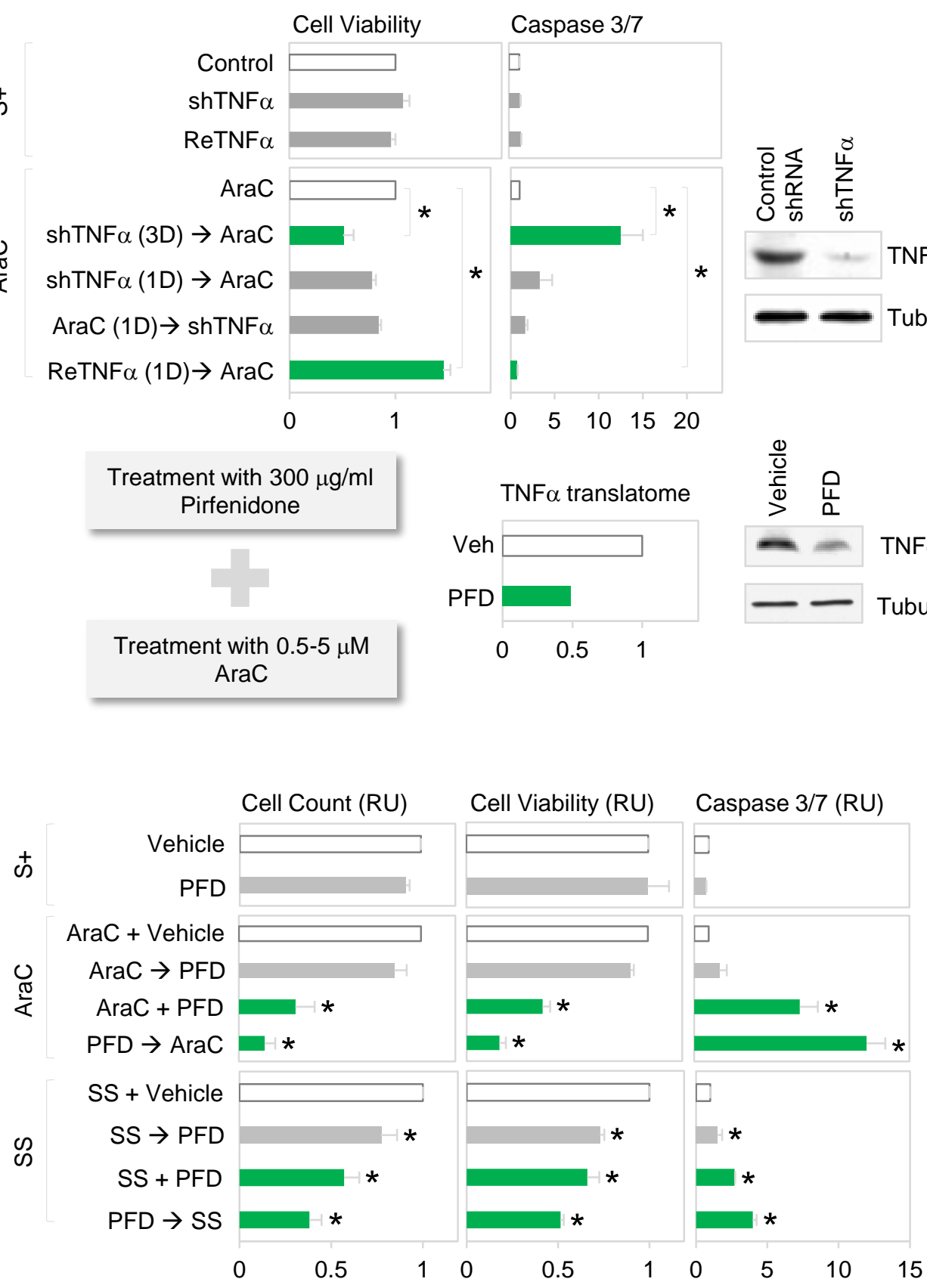

AraC (1D) $\rightarrow$ shTNF $\alpha$

$\begin{array}{lllll}0 & 5 & 10 & 15 & 20\end{array}$

Treatment with $300 \mu \mathrm{g} / \mathrm{ml}$ Pirfenidone

Treatment with 0.5-5 $\mu \mathrm{M}$ AraC
G
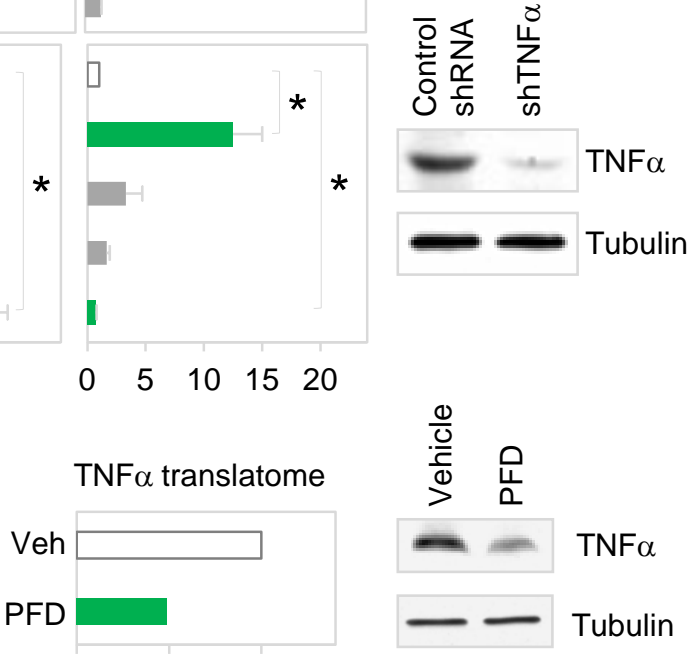

$\mathrm{H}$
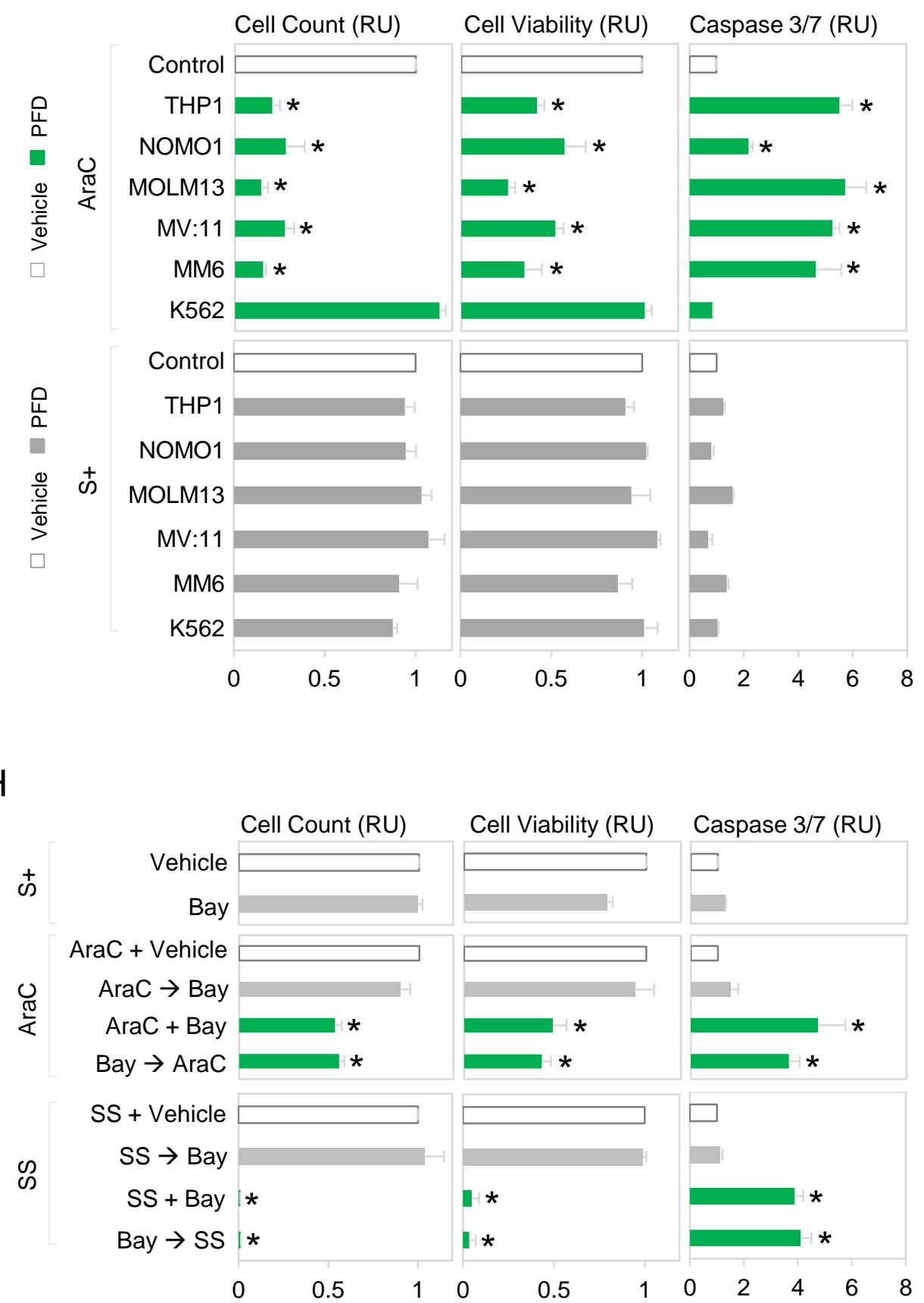

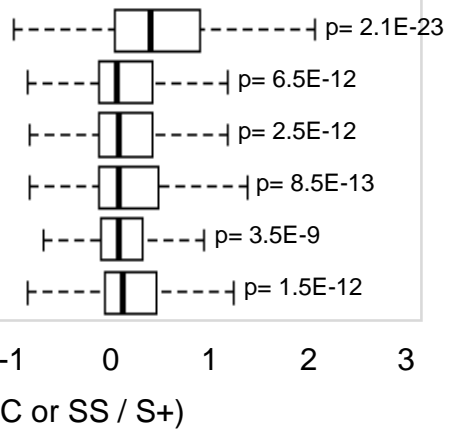

S+ SS AraC

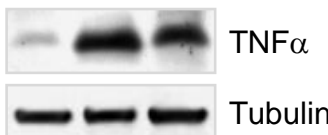


A

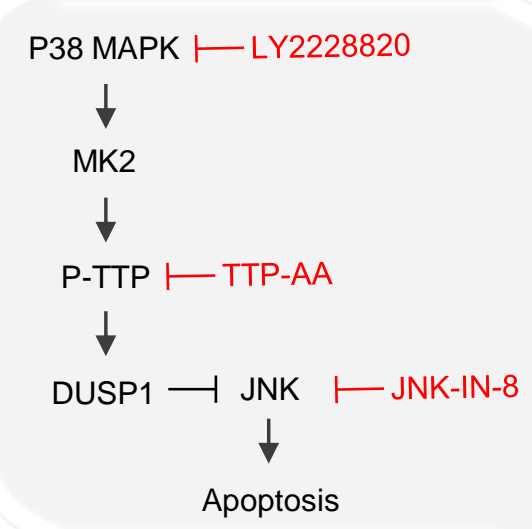

E

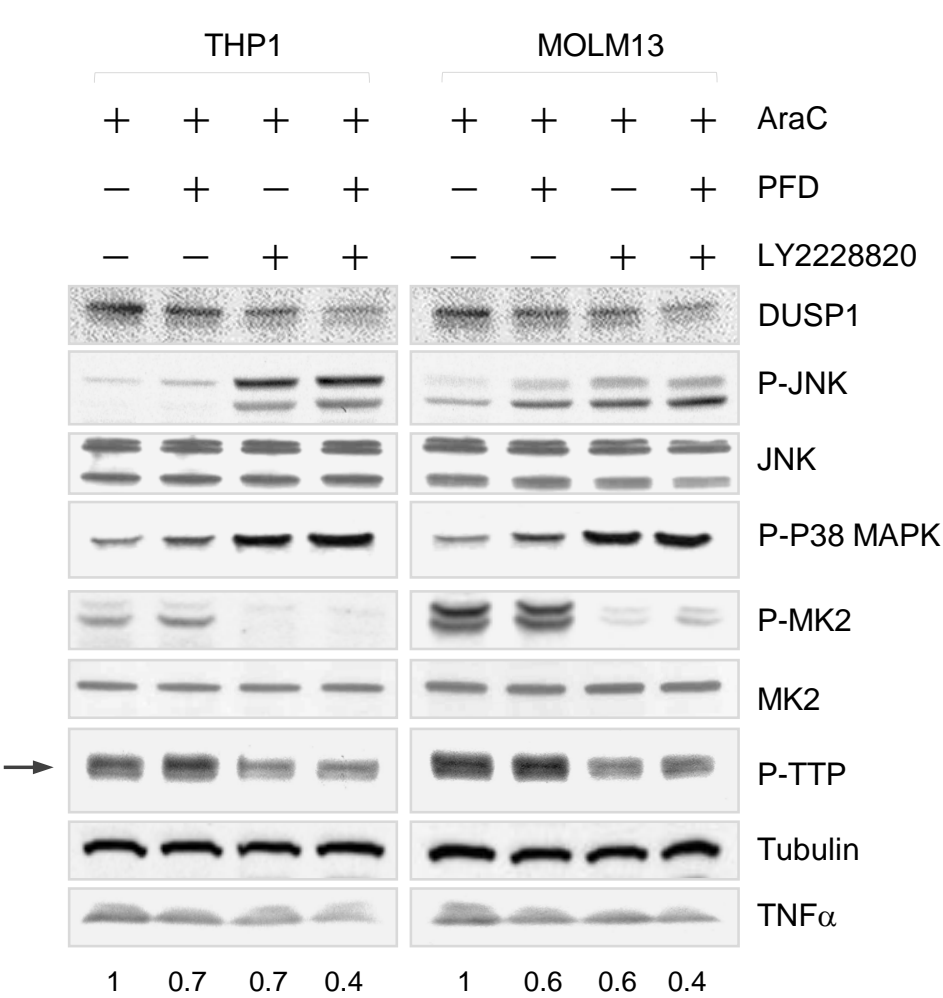

B

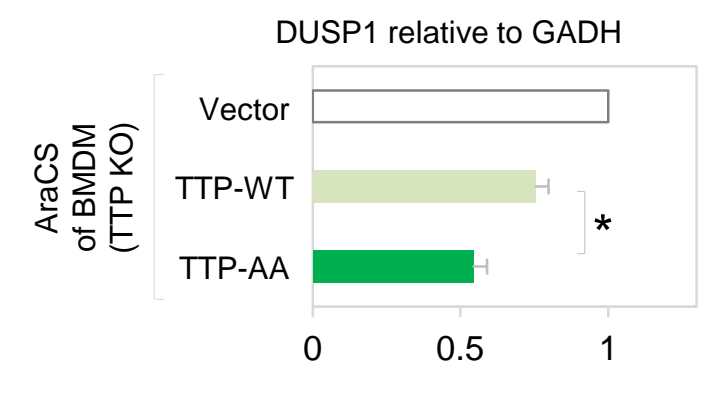

C

AraCs

of BMDM

(TTP KO)

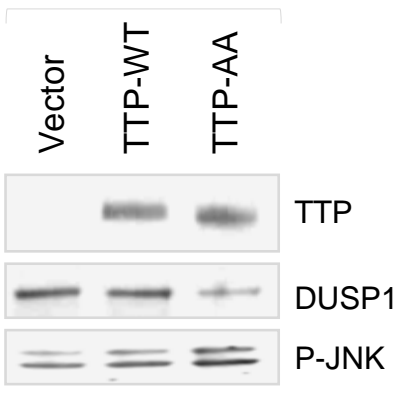

D

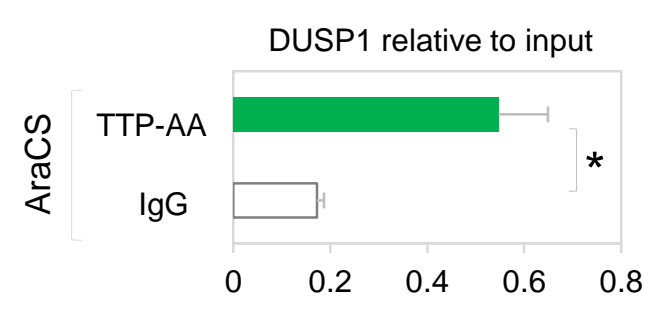

F

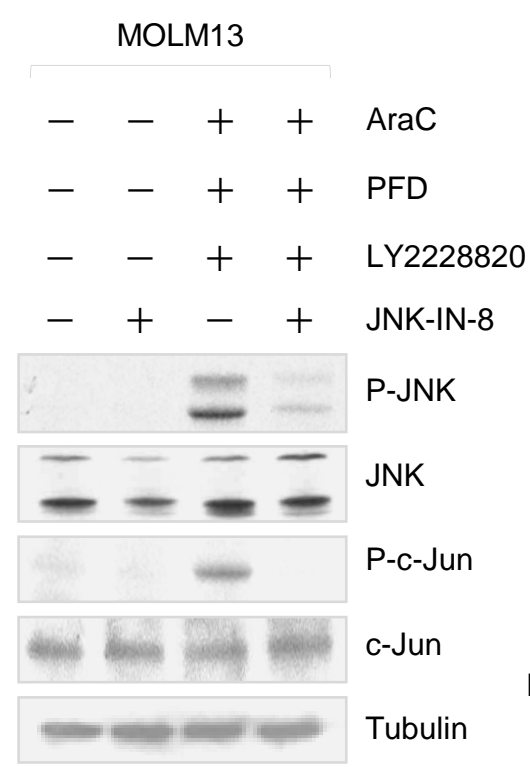

MOLM13

$-\quad++\operatorname{AraC}$

$-\quad++$ PFD

$-\quad+\quad+\quad$ LY2228820

-+-+ JNK-IN-8
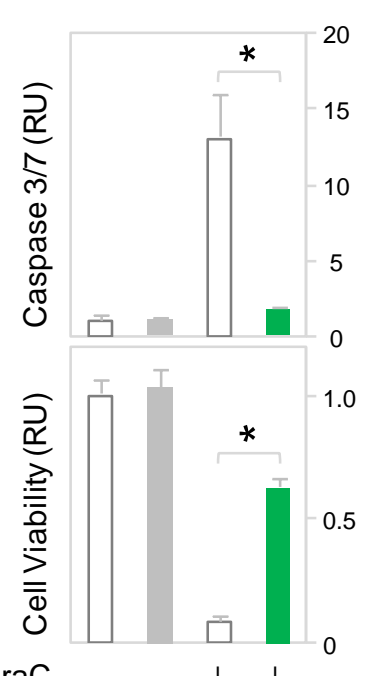

AraC --++

LY2228820 - - + +

PFD $-\quad++$

JNK-IN-8 - + - +

MOLM13 
A

B

PLA therapy

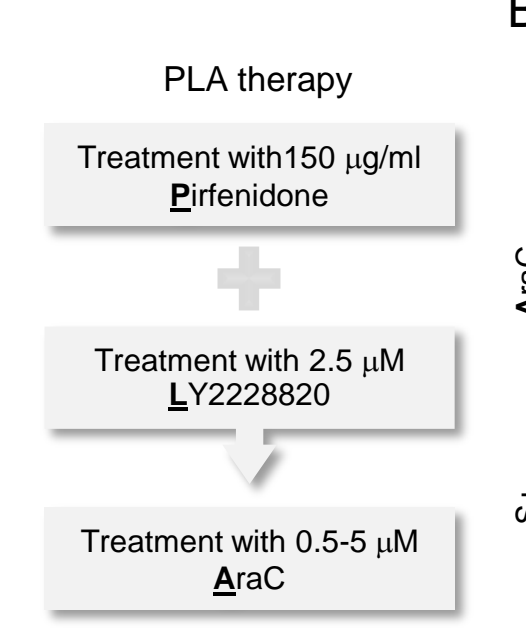

C

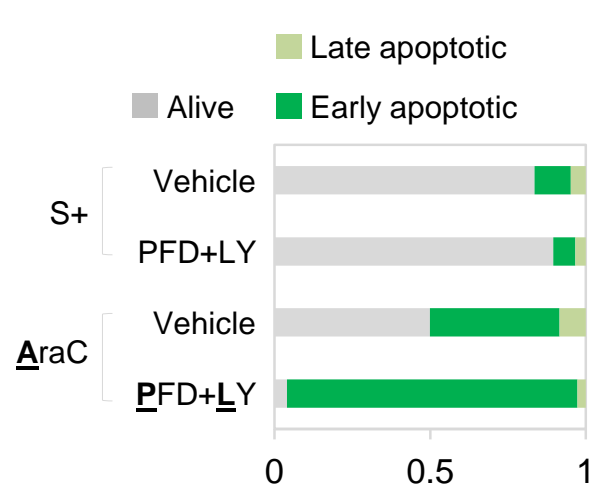

D
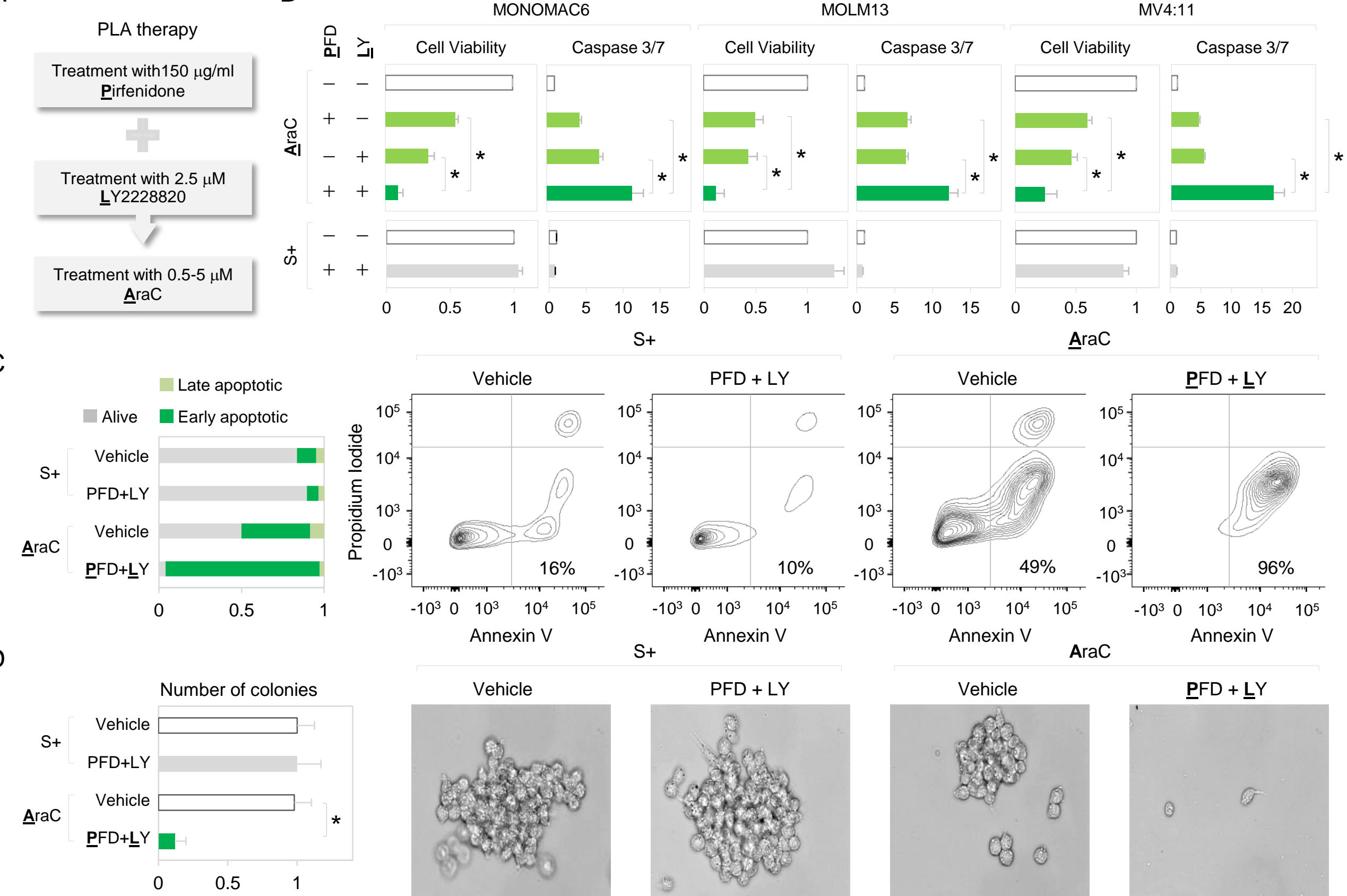

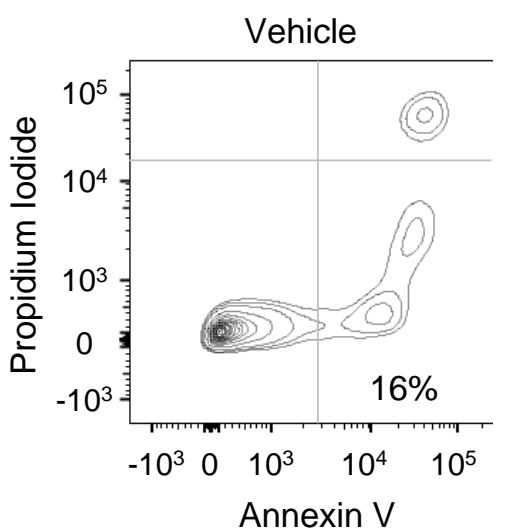

Vehicle

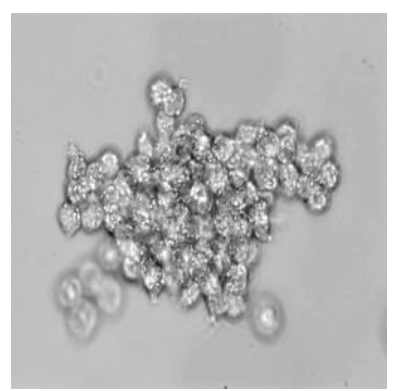

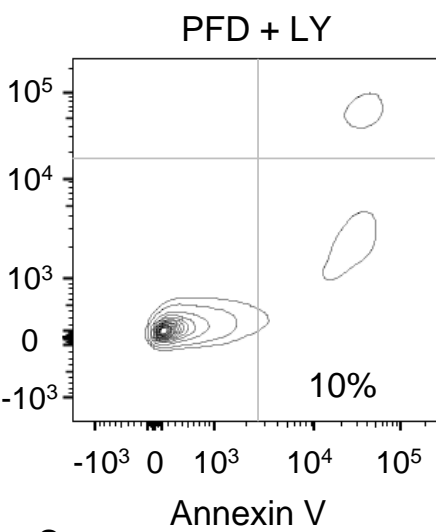

S+

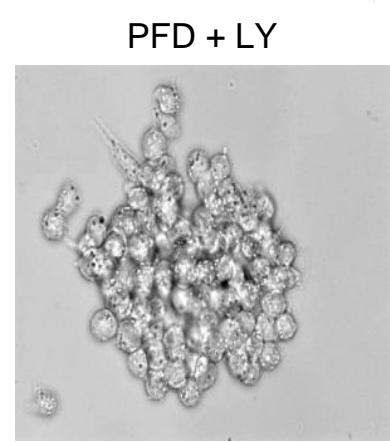

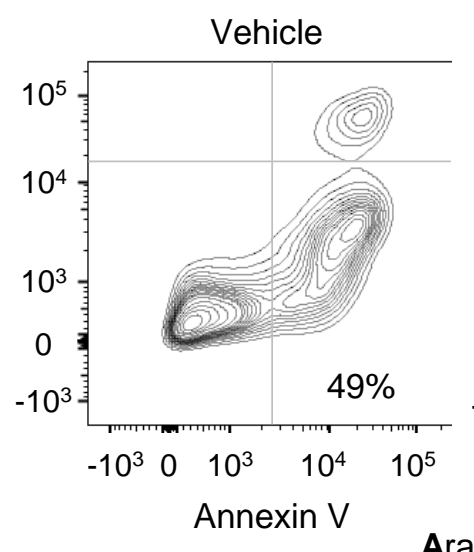
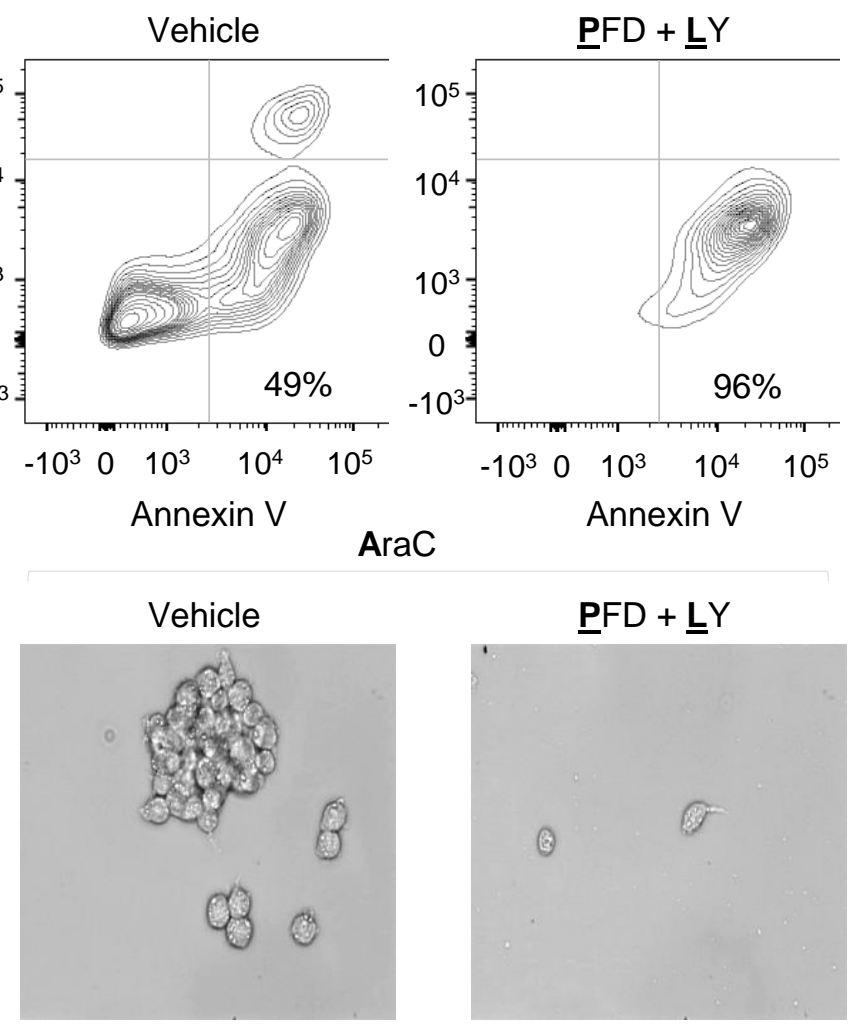
A $\quad 1.6$ bioRxiv preprint doi: https://doi.org/10.1101/418715; this version posted May 3, 2019. The copright holder for this preprint (which was not
certified by peer review) is the author/funder, who has granted bioRxiv a license to display the pleprint in perpetuity. It is made available under

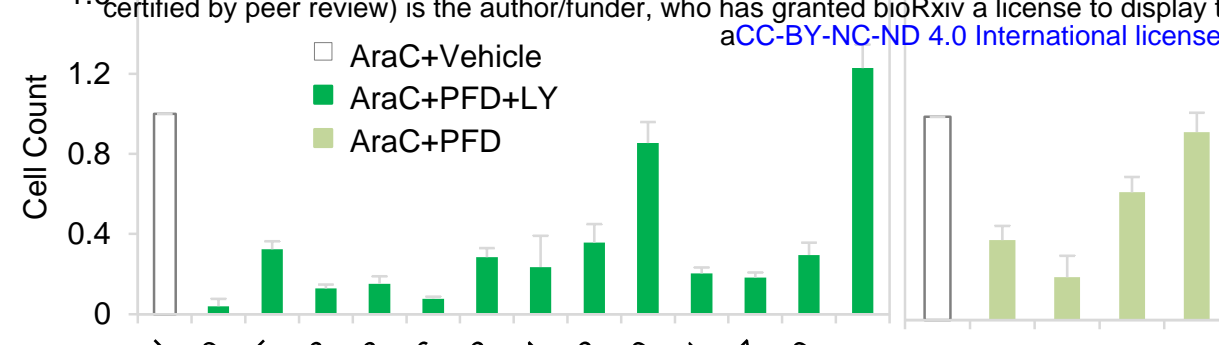

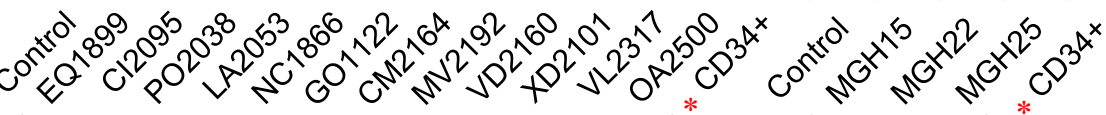

AML patients

AML patients

C
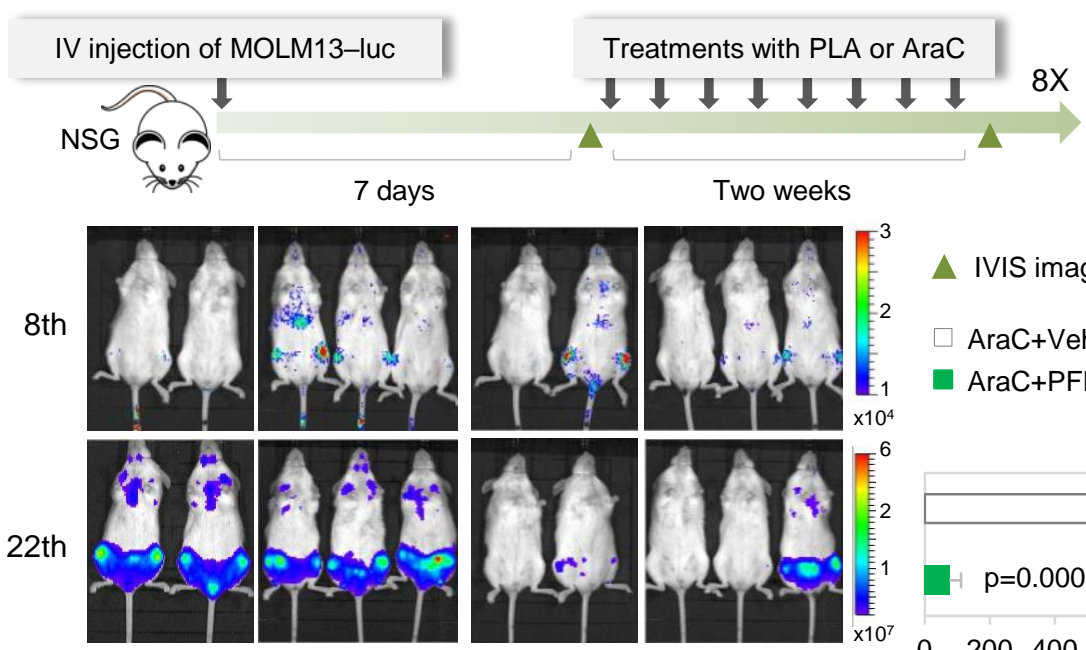

AraC + Vehicle

E
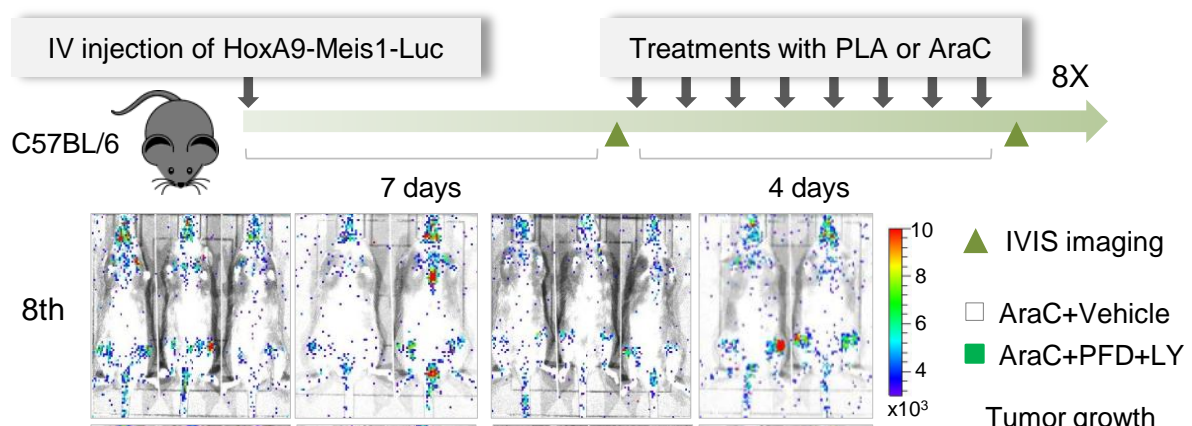

15 th
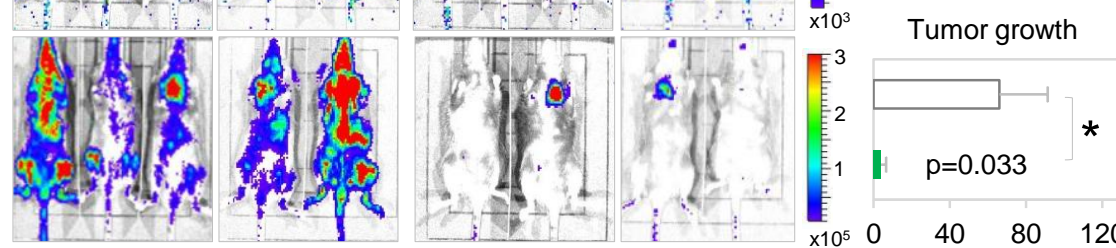

AraC + Vehicle

$\mathrm{AraC}+\mathrm{PFD}+\mathrm{LY}$

G
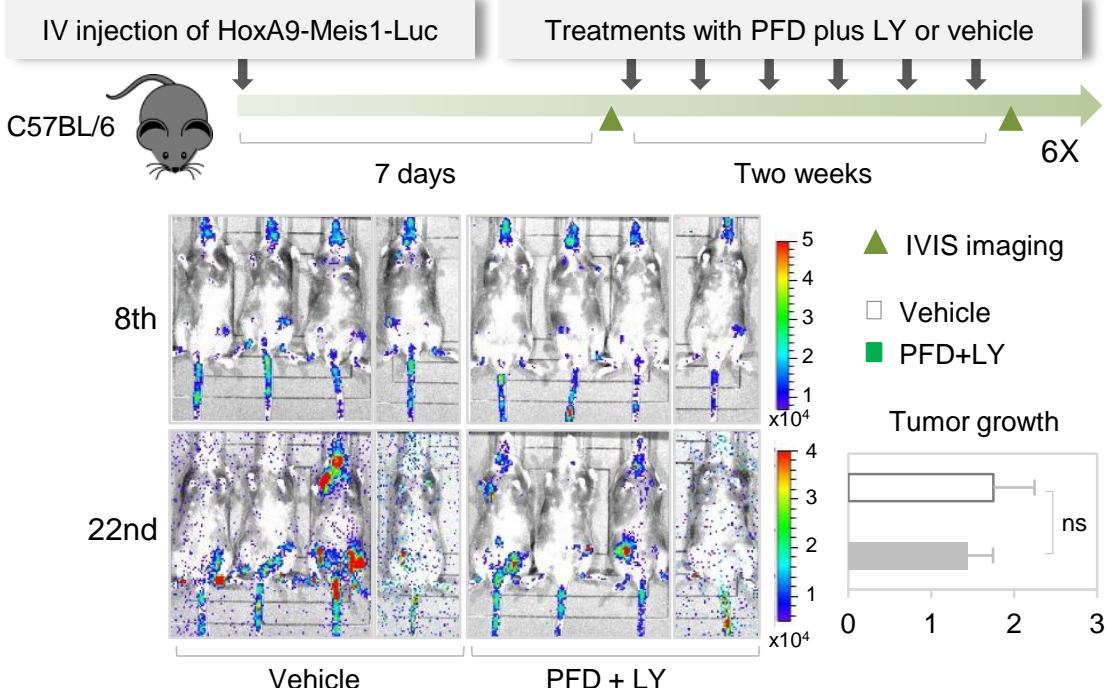

HoxA9-Meis1

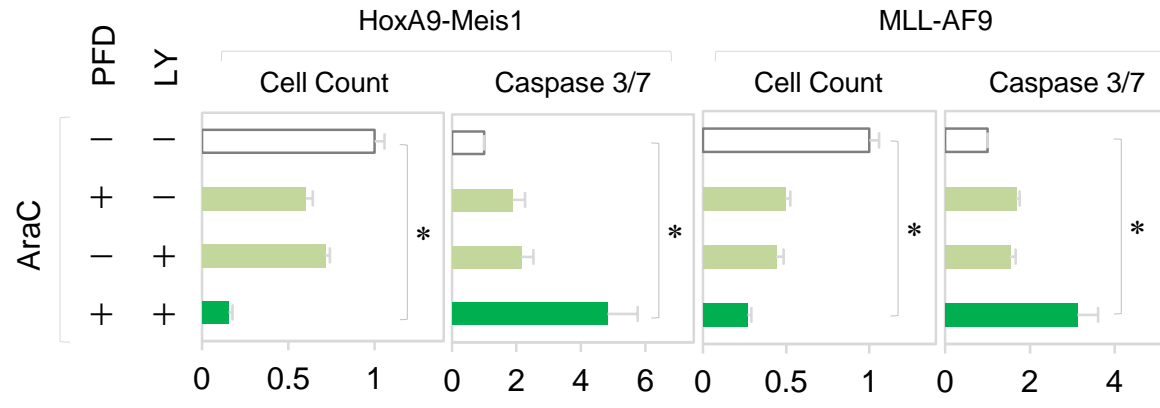

D
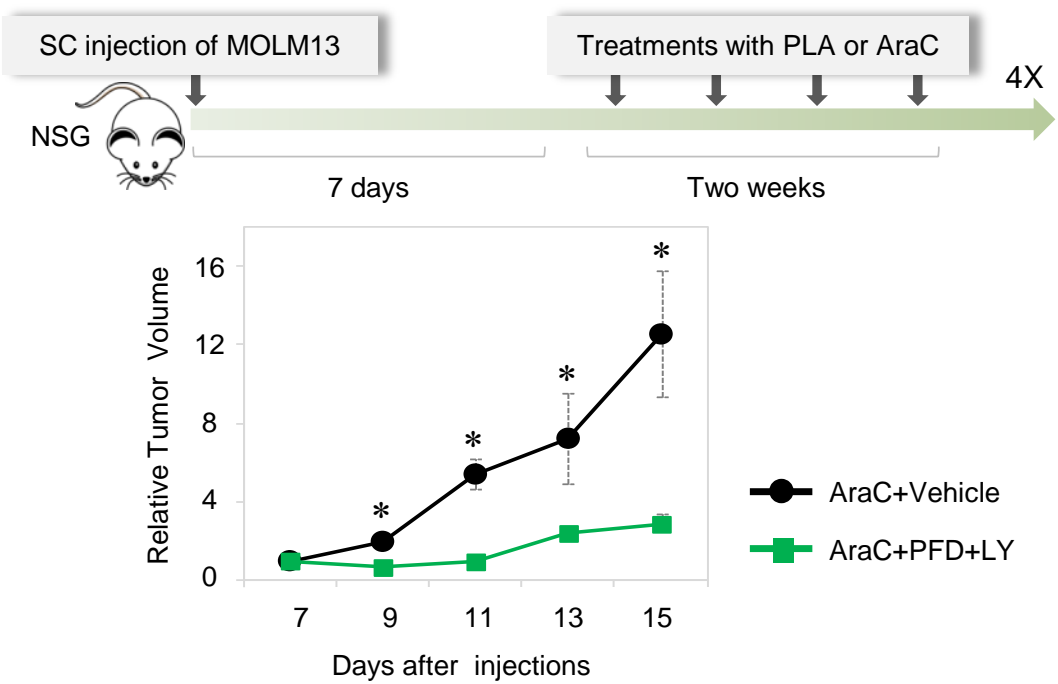

F
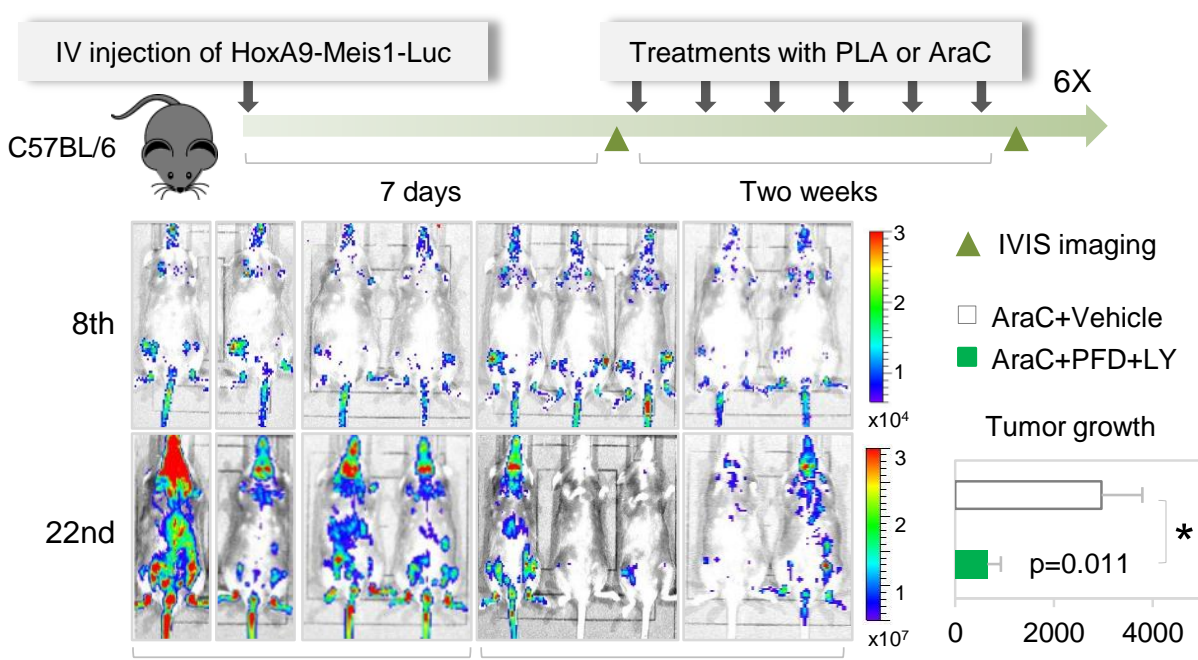

AraC + Vehicle $\quad$ AraC + PFD + LY

$\mathrm{H}$
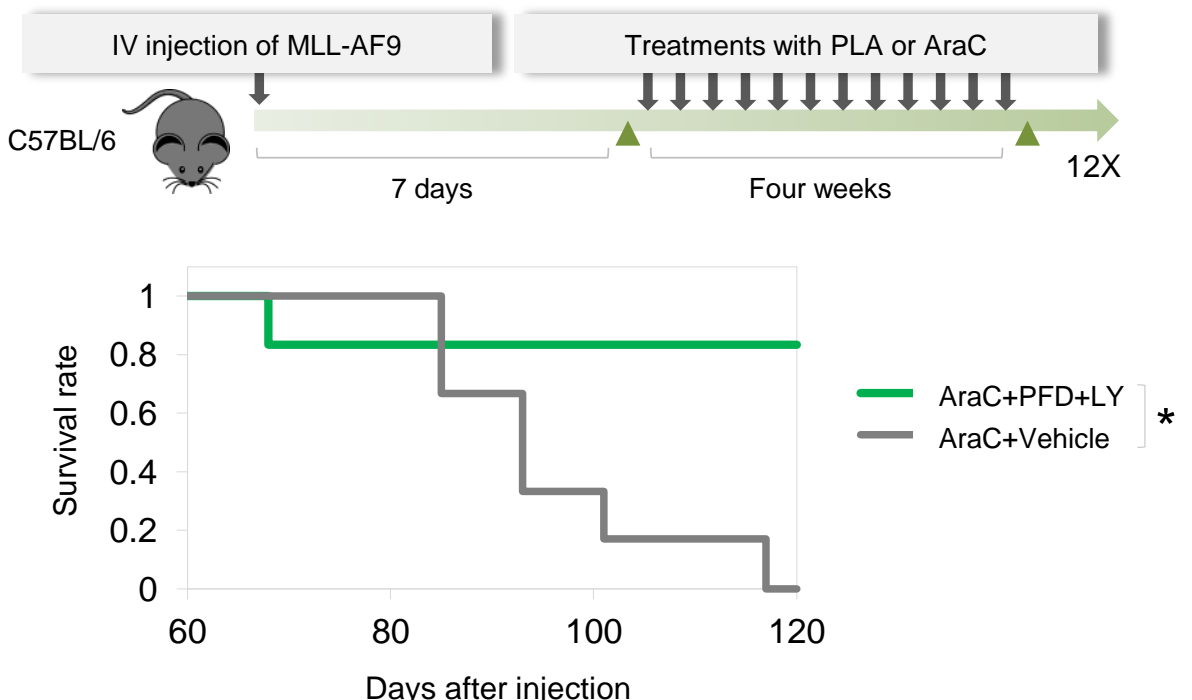